\title{
»Ein echter Naturforscher von heute hat eine tiefe Verachtung gegen $>$ Literatur $\ll$
}

\section{Zeitgemäße Wechselbeziehungen zwischen Physik, Literatur und Philologie im frühen 20. Jahrhundert}

\author{
Magdalena Gronau • Martin Gronau
}

Angenommen: 20. Oktober 2021 / Online publiziert: 24. November 2021

(C) Der/die Autor(en) 2021

Zusammenfassung Der vorliegende Aufsatz beleuchtet die komplexen Wechselbeziehungen zwischen Physik, Literatur und Philologie in der ersten Hälfte des 20. Jahrhunderts. Ausgehend von einem Brief des österreichischen Physikers Erwin Schrödinger an Ernst Cassirer gehen wir der Frage nach, inwiefern die prominenten deutschsprachigen Vertreter der aufstrebenden theoretischen Physik nicht nur literarische Texte und Inhalte verarbeiteten, sondern in ihrer zutiefst philologisch geprägten Publikations- und Arbeitspraxis auch textwissenschaftlich operierten. Lassen sich in den nachgelassenen und publizierten Schriften von Physikern wie Schrödinger neue Hinweise darauf finden, dass die »Macht der Philologie « noch weit ins Jahrhundert der Physik nachwirken, ja Philologie vielleicht sogar als Wissensmodell in der theoretischen Physik fungieren konnte?

Magdalena Gronau $(\bowtie) \cdot$ Martin Gronau

Neuere Deutsche Literaturwissenschaft, Universität Erfurt, Nordhäuser Straße 63, 99089 Erfurt, Deutschland

E-Mail: magdalena.gronau@uni-erfurt.de 


\title{
»Ein echter Naturforscher von heute hat eine tiefe Verachtung gegen $>$ Literatur $\ll$
}

Contemporary interrelations between physics, literature, and philology in the early 20th century

\begin{abstract}
This essay examines the complex interrelations between physics, literature, and philology in the first half of the 20th century. Starting from a letter of the Austrian physicist Erwin Schrödinger to Ernst Cassirer, we investigate how theoretical physicists in the German-speaking world referred to literary texts and contents. Beyond that, we focus on traces of philological working and publication practices in the works of the physicists. To what extent and under what conditions could philology function as an inevitable knowledge model for the modern natural sciences?
\end{abstract}

\section{I.}

\section{Ein Brief}

Ernst Cassirer sandte seine 1937 im Göteborger Exil publizierte Studie Determinismus und Indeterminismus in der modernen Physik ${ }^{1}$ an zahlreiche Atomphysiker. Koryphäen wie Max Born, Werner Heisenberg, Niels Bohr sowie dessen ehemaliger Schüler Hendrik Kramers, Max von Laue oder Albert Einstein haben jeweils ein Exemplar erhalten. In den überlieferten Antwortschreiben der Naturwissenschaftler finden sich neben fallweise wenig substanziellen Dankesschreiben ${ }^{2}$ auch umfangreiche Kommentare, welche das Interesse der Physiker an der Rezeption ihrer Forschungen jenseits der Fachgrenzen belegen. ${ }^{3}$ Ein Brief sticht aus diesen interdisziplinären Korrespondenzen besonders hervor, verfasst am 09. Mai 1937 vom Nobelpreisträger Erwin Schrödinger, ${ }^{4}$ der nach der Machtergreifung der National-

\footnotetext{
1 Ernst Cassirer, Determinismus und Indeterminismus in der modernen Physik. Historische und systematische Studien zum Kausalproblem, Göteborg 1937. Die Werke Ernst Cassirers werden im Folgenden aus der (digitalen) Ausgabe unter Angabe der Band- und Kapitelnummer zitiert: Ernst Cassirer, Gesammelte Werke. Hamburger Ausgabe, hrsg. Birgit Recki, Hamburg 2009.

2 Vgl. dazu den an Ernst Cassirer gerichteten Brief (24.03.1937) von Werner Heisenberg, der sich »über viele Formulierungen in Ihrem Buch sehr gefreut « hat und Cassirers Buch als Beleg dafür sieht, dass nach dem für die kommenden zehn bis fünfzehn Jahre in Aussicht gestellten Abschluss der Atomphysik »viel Wichtiges auf diesem Grenzgebiet von Philosophie und Naturwissenschaft « zu erfahren sei. Zit. nach Ernst Cassirer, Nachgelassene Manuskripte und Texte. Band 18: Briefwechsel, DVD-ROM-Edition, hrsg. John Krois, Hamburg 2009, hier: Nr. 1141.

3 Hendrik Kramers etwa schreibt eine zehnseitige Antwort (26.03.1937). Max von Laue listet in insgesamt drei Briefen (vom 23.03., 26.03. und 04.04.1937) eine Reihe von Kritikpunkten auf und ergänzt diese um eigene Denkanstöße. Albert Einstein äußert sich wiederum anerkennend, ist aber mit Blick auf die Vollständigkeit der Quantenphysik skeptisch (16.03.1937). Auch Max Born antwortet konkret und detailliert (19.03.1937). Vgl. Cassirer (Anm. 2), hier: Nr. 1144, 1140, 1143, 1146, 1137, 1138.

4 Brief von Erwin Schrödinger an Ernst Cassirer (09.05.1937), zit. nach Karl von Meyenn (Hrsg.), Eine Entdeckung von ganz außerordentlicher Tragweite. Schrödingers Briefwechsel zur Wellenmechanik und zum Katzenparadoxon, Berlin, Heidelberg 2011, hier: 588-590.
} 
sozialisten seinen reputierlichen Berliner Lehrstuhl für theoretische Physik aufgegeben und Deutschland Richtung Oxford verlassen hatte. Schrödinger - in der wissenschaftshistorischen Rückschau oft als einzelgängerischer Querdenker der damals noch überschaubaren Zunft charakterisiert ${ }^{5}$ und wechselweise als Vertreter einer konservativen Anschaulichkeitsphysik, als Ästhet, Dichter und transdisziplinärer Universalist wahrgenommen ${ }^{6}$ - findet gegenüber dem Ordinarius für Philosophie kritischere Worte als seine Fachgenossen. Er selbst könne dessen Studie eigentlich nichts Nennenswertes hinzufügen, oder eher: angesichts der Flut an Beiträgen zur Philosophie der Quantenphysik wolle er das gar nicht. Mit Komplimenten hält Schrödinger sich zurück, ja bewegt sich beinahe an der Grenze zur Unhöflichkeit und scheut sich nicht, mit dem Kollegen harsch ins Gericht zu gehen: Recht unverblümt bringt er etwa seinen Unmut darüber zum Ausdruck, dass »gerade einem Philosophen das unendlich angeschwollene Tam-tam der modernen Quantenphysik « eigentlich offensichtliche Übereinstimmungen bestimmter Deutungsansätze verdecken würde. Der durchwegs raue Ton verwundert umso mehr, als die beiden Wissenschaftler sich während ihres zeitgleichen Exilaufenthalts an der britischen Eliteuniversität persönlich begegnet sind und sich dort kollegial-freundschaftlich über das Sujet ausgetauscht haben dürften. ${ }^{7}$

Im Zentrum des dreiseitigen Typoskripts steht eine polemische Belehrung Schrödingers über die angeblich ablehnende Haltung der modernen Naturwissenschaftler gegenüber den »Kategorientafeln älterer Philosophen, die noch keine Ahnung hatten von dem, was aus der Erkenntnis heute geworden ist «. Dem zeitgenössischen Physiker scheinen solche »feinen Begriffsschemata«, oder, wie er Cassirer gegenüber maliziös spezifiziert, »jedes Zitat z.B. aus Kant«, »fast so veraltet und unnütz wie ein Adressbuch aus den achziger [sic] Jahren«. Zur Veranschaulichung dieser angeblich traditionsskeptischen Haltung bemüht Schrödinger ein recht martialisches Bild: »Wir haben das Gefühl, in der vordersten Linie zu stehen, einen, sagen wir, Tank-, Flugzeug- und Gaskrieg zu führen, und können nur schwer überzeugt werden, dass wir aus den strategischen Werken jener, die mit Schwert und Speer, mit Helm und Panzer zu Felde zogen, für unsere heutigen Aufgaben viel Nutzen ziehen können. « ${ }^{8}$ Die in der wir-Form, also im Namen der »echte[n] Naturforscher von heute«, be-

\footnotetext{
5 So bezeichnet z.B. der Wissenschaftshistoriker Michael Eckert Schrödinger als »etwas isolierten Privatdozenten«, der »abseits der atomtheoretischen Zentren« arbeitete. Michael Eckert, Die Atomphysiker. Eine Geschichte der theoretischen Physik am Beispiel der Sommerfeldschule, Wiesbaden 1993, hier: 60, 97.

6 Vgl. z.B. Hans Ulrich Gumbrecht, Robert Pogue Harrison, Michael R. Hendrickson, Robert B. Laughlin (Hrsg.), Geist und Materie. Zur Aktualität von Erwin Schrödinger, Frankfurt a.M. 2008.

7 Thomas Ryckman, »A Retrospective View of >Determinism and Indeterminism< in Modern Physics «, in: Tyler J. Friedman, Sebastian Luft (Hrsg.), The Philosophy of Ernst Cassirer. A Novel Assessment, Berlin, Boston 2015, 65-102, hier: 83.

8 Und weiter: »Alles muss doch aus der Sache selbst folgen. Was ein anderer vorher darüber gedacht oder geschrieben haben mag, ist schon dadurch suspekt, dass, wenn es auch nur 10 Jahre zurückliegt, ihm sehr wesentliche Kenntnisse zur Beurteilung des Falles abgegangen haben müssen. Selbst einen primitiven, einfachen Rechengang macht man sich lieber selbst neu, anstatt erst lang Bände zu wälzen, selbst wenn man weiss, dass die Überlegung schon vorliegen muss. Erstens versteht man die Sache dann wirklich, zweitens vermeidet man es so, etwaige naive Irrtümer mitzumachen, die seither aufgedeckt worden sind. Daher rührt unsere Abneigung gegen jedes Zitat z.B. aus Kant oder gegen die Verwendung von Begriffsschemata, die aus seiner oder irgend einer ähnlichen Hexenküche stammen.« Schrödinger an Cassirer (Anm. 4).
} 
kundete Abneigung gegenüber der »Literatur « und allem, was aus einer »ähnlichen Hexenküche « stamme, wirkt auf den ersten Blick wie ein Zeugnis der nicht einmal zwanzig Jahre später virulent werdenden Kontroverse um die »Two Cultures «. ${ }^{9}$ Der Physiker scheint sich selbst, seine Disziplin und ihre am laufenden Band neues und verwertbares Wissen hervorbringenden Techniken von den langlebigeren, mit hohem kulturellen Kapital ausgestatteten geisteswissenschaftlich-literarischen Wissensformationen abzugrenzen. Entgegen diesem ersten Deutungsimpuls möchten wir das schroffe Dankesschreiben Schrödingers an Cassirer jedoch nicht zum Anlass nehmen, in anachronistischer Weise die modernen »Science Wars « in die 1930er-Jahre zurück zu projizieren. Vielmehr wollen wir hinter die vermeintlich klar gezogenen Frontlinien blicken und am Beispiel Schrödingers gängige Diskursstränge und interdisziplinäre Berührungshorizonte ausleuchten, um so ein differenziertes Bild des Beziehungsgefüges von Physik und Literatur im frühen 20. Jahrhundert zu entwickeln. Nicht zuletzt soll geklärt werden, wie zeit- oder vielleicht auch unzeitgemäß die von Schrödinger so ostentativ postulierte »Verachtung gegen die Literatur « unter den »echten Naturforscher[n] von heute« denn nun wirklich war.

II.

\section{Schrödinger und die Literatur}

Wirft man einen auch nur oberflächlichen Blick in die publizierten und nachgelassenen Schriften Erwin Schrödingers und seiner Fachkollegen, so regen sich schnell Zweifel an der behaupteten a-literarischen Gesinnung der damaligen Physik. Schrödingers pauschalisierende Bemerkungen erstaunen umso mehr, als er selbst seine Arbeiten nicht nur mit damals selbstverständlichem bildungsbürgerlichen Kanon-

\footnotetext{
9 Silke Jakobs, »Selbst wenn ich Schiller sein könnte, wäre ich lieber Einstein«. Naturwissenschaftler und ihre Wahrnehmung der »zwei Kulturen«, Frankfurt a.M., New York 2006. Vgl. einführend zur Two Cultures-Debatte auch Nicolas Pethes, »Literatur- und Wissenschaftsgeschichte. Ein Forschungsbericht«, Internationales Archiv für Sozialgeschichte der deutschen Literatur 28 (2007), 181-231, hier: v.a. 186-191.

10 Derartige auch von Schrödinger angewandte Strategien sind z.B. im Fall von Werner Heisenbergs extensiver Bezugnahme auf Goethe, die u.a. in einem Vortrag vor der Goethe-Gesellschaft in Weimar gipfelte, ausführlich erforscht. Werner Heisenberg, »Die Goethe'sche und die Newton'sche Farbenlehre im Lichte der modernen Physik«, in: Ders., Gesammelte Werke. Abteilung C: Allgemeinverständliche Schriften. Band I: Physik und Erkenntnis 1927-1955, hrsg. Walter Blum, Hans-Peter Dürr, Helmut Rechenberg, München 1984, 146-160; Werner Heisenberg, »Das Naturbild Goethes und die technisch-naturwissenschaftliche Welt«, in: Ders., Gesammelte Werke. Abteilung C: Allgemeinverständliche Schriften. Band II: Physik und Erkenntnis 1956-1968, hrsg. Walter Blum, Hans-Peter Dür, Helmut Rechenberg, München 1984, 394-409. Vgl. zu Heisenbergs Verhältnis zu Goethe eher identifikatorisch Helmut Rechenberg, » Goethe hat ihn durch sein ganzes Leben begleitet<. Werner Heisenbergs Auseinandersetzung mit Goethes Naturbild«, Goethe-Jahrbuch 120 (2003), 277-291. Kritisch dazu: René Jacques Baerlocher, »Bemerkungen zu Werner Heisenbergs Goethebild«, Goethe-Jahrbuch 122 (2005), 243-262. Vgl. außerdem Cathryn Carson, Heisenberg in the Atomic Age. Science and the Public Sphere, New York 2010; Peter Huber, Naturforschung und Meßkunst. Spuren Goethescher Denkart in der frühen Quantentheorie, Hamburg 2000; Alexandra Skowronski, »Heisenberg und Goethe - Physik und Dichtung. Strategien naturwissenschaftlicher und bildungsbürgerlicher Selbstdarstellung am Beispiel von Werner Heisenbergs Goethe-Vorträgen (1941-1967)«, Scientia Poetica 15 (2011), 252-296.
} 
wissen garnierte, ${ }^{10}$ sondern sich immer wieder als Feingeist mit umfassenden literarisch-philosophischen Interessen auswies, der umgekehrt auch von zeitgenössischen Literaten rezipiert wurde - insbesondere was sein etwa ab 1940 entstandenes, eher essayistisch-interdisziplinär angelegtes Spätwerk betrifft. ${ }^{11}$

Berührungspunkte zwischen Physik und Literatur, die von losen Affinitäten über wechselseitige Rezeptionen bis hin zu personell nachverfolgbaren Kontakten reichen, sind in der ersten Hälfte des 20. Jahrhunderts jedenfalls allgegenwärtig. ${ }^{12}$ Oft reichen sie weit über die im Briefwechsel mit Cassirer thematisierten philosophischen und epistemologischen Implikationen der Quantentheorie hinaus. So ist beispielsweise Schrödingers Lektüre der physikalisch recht gehaltvollen Bekenntnisse des Hochstaplers Felix Krull verbrieft, aus denen er in seinen Notizbüchern einige Passagen exzerpiert hat. ${ }^{13}$ Thomas Mann wiederum, dessen Bewunderung für zeitgenössische Forscher wie Albert Einstein sogar fotografisch dokumentiert ist, ${ }^{14}$ war nur einer von vielen Schriftstellern, die sich intensiv mit den wissenschaftlichen Revolutionen ihrer Zeit auseinandersetzten und dabei auch in Lektüre-Kontakt mit Schrödinger geraten konnten. ${ }^{15}$ So vermerkte etwa Robert Musil, seinerseits bekanntlich einschlägig vorgebildet, zufrieden in seinem Tagebuch: »Ich lese - nach Abschluß des I Bdes. MoE. - in Der Koralle (Dez. 1929) eine Plauderei von Erwin Schrödinger Mitgl. d. Preuss. Ak. d. Wiss. über >Das Gesetz der Zufälle<. Danach ist die Frage Kausal- oder statistisches Gesetz jetzt sehr aktuell. $\ll^{16}$ Die Notiz sowie die anschließende Abschrift einer Passage des Artikels könnte man als weiteren Beleg für Musils mathematisch-physikalische Neigungen und sein literarisches Gespür

\footnotetext{
${ }^{11}$ Darunter wären etwa Schrödingers Vortragsreihe Nature and the Greeks, sein breit rezipiertes Büchlein What is Life? oder der eher philosophische Band Geist und Materie zu fassen: Erwin Schrödinger, Nature and the Greeks. Shearman Lectures, delivered at University College, London, on 24, 26, 28, and 31 May 1948, Cambridge u.a. 1954; Erwin Schrödinger, What Is Life? The Physical Aspect of the Living Cell, Based on Lectures delivered under the auspices of the Institute at Trinity College, Dublin, in February 1943, Cambridge u.a. 1944; Erwin Schrödinger, Geist und Materie, Braunschweig 1965. Vgl. auch Elisabeth Emter, Literatur und Quantentheorie. Die Rezeption der modernen Physik in Schriften zur Literatur und Philosophie deutschsprachiger Autoren (1927-1970), Berlin, New York 1995, hier: 314-328, die sich intensiv mit Wolfgang Koeppens Rezeption von Schrödingers What is Life? auseinandersetzt.

12 Vgl. dazu v.a. Elisabeth Emters umfangreiche Analysen, Emter (Anm. 11).

13 Österreichische Zentralbibliothek für Physik, Wien, Nachlass Erwin Schrödinger (o:143722), Sign. W33-786. Dokumente aus dem Nachlass werden im Folgenden zitiert als: ÖZB (o:1437222), Sign. x. Biografische Parallelen zwischen den beiden Nobelpreisträgern dürften sich nicht auf die jeweils sehr intensive Schopenhauer-Lektüre und ihre breit gestreuten Interessen beschränken. Eine vor wenigen Jahren in Arosa gezeigte Ausstellung band die Lebensschicksale der Exilanten Thomas Mann und Erwin Schrödinger unter dem Titel »Lebenswende in Arosa« zusammen, vgl. http://www.arosa-museum.ch/ausstattung/ 542074a6ba0a47903/index.htm (07.10.2021).

${ }^{14}$ Bekannt ist ein im Nachlass Manns verwahrtes Foto der beiden Nobelpreisträger, das Thomas Mann mit der Bildunterschrift »So ganz einfach neben dem? Mir schwindelt.« versehen hat. Malte Herwig, Bildungsbürger auf Abwegen. Naturwissenschaft im Werk Thomas Manns, Frankfurt a.M. 2004, Abbildungen.

${ }^{15}$ Eine nicht unwesentliche Rolle spielten dabei auch Mittlerfiguren wie etwa Günther Anders, der, an der Schnittstelle von Literatur und Wissenschaft(skritik) positioniert, für die Anti-Atomtod-Bewegung prominente Personen beiderlei Sphären zu gewinnen suchte. Vgl. dazu auch Clemens Özelt, Literatur im Jahrhundert der Physik. Geschichte und Funktion interaktiver Gattungen (1900-1975), Göttingen 2018, 344-350.

${ }^{16}$ Zit. nach Emter (Anm. 11), hier: 106. Vgl. Erwin Schrödinger, »Das Gesetz der Zufälle. Der Kampf um Ursache und Wirkung in den modernen Naturwissenschaften«, Die Koralle 5 (1929), 417-418.
} 
für das wissenschaftsphilosophische Klima der 1920er-Jahre lesen. Mit Blick auf die hier diskutierten Fragen ist der Kommentar des Schriftstellers indes in erster Linie als Hinweis auf eine gewisse Stilsicherheit Schrödingers im Umgang mit neuen Formaten wie den reichhaltig bebilderten, populärwissenschaftlichen Illustrierten von Bedeutung: ${ }^{17}$ Die versierte Bespielung moderner medialer Formen erweist sich, gerade bei prominenten, manchmal ikonischen Forscherpersönlichkeiten wie Schrödinger, ${ }^{18}$ als wichtiger Faktor, der das Interesse einer fachfremden Öffentlichkeit an disziplinären Entwicklungen und Debatten positiv beeinflusste. Schrödingers journalistisch-schmissige, in Zeitschriften wie der Koralle, den Naturwissenschaften oder der Uhu-Umschau veröffentlichte Artikel zur Doppelnatur des Lichts, zu Elektrizität oder Kausalität und Zufall in der Physik sind in dieser Hinsicht durchaus wissensvermittelnden Ansätzen der jüngeren Vergangenheit vergleichbar, wie sie sich etwa bei Stephen Hawking, Anton Zeilinger oder, ganz aktuell, dem Virologen Christian Drosten festmachen lassen. Dass Schrödinger dabei gerne die Techniken der Literatur in den Dienst der Didaxe stellte, wurde und wird immer wieder auch von Fachkollegen hervorgehoben, beispielsweise vom Mathematiker Detlef Dürr, der Schrödingers vielzitierten Aufsatz zur »Gegenwärtigen Situation in der Quantenmechanik « als »literarisch « einstuft: »Formeln findet man [dort] nur ab und an «. ${ }^{19}$ Der hinsichtlich seiner Auslegung der neuen Physik als konservativ geltende, mitunter sogar als $\gg$ regressiv $\ll^{20}$ abgekanzelte Schrödinger entpuppt sich als moderner Wissenschaftskommunikator, der mit seinen flott geschriebenen, kokettierenden ${ }^{21}$ Zeitschriften-Artikeln auf den Zug der Zeit aufgesprungen ist.

In seinem konkreten Umgang mit literarischen Werken und Formen ist Schrödinger hingegen kaum als Trendsetter zu bezeichnen. Viele der überlieferten Zeugnisse lassen sich einem weit verbreiteten Modell literarisch affizierter naturwissenschaftlicher Selbstdarstellung zuordnen, an das selbst heutige Wissenschaftler/innen fallweise noch anknüpfen. ${ }^{22}$ So verarbeitete Schrödinger in seinen philosophischen Reflexionen, in Briefen und in nachgelassenen Notizen spätestens seit den 1920erJahren ein Sammelsurium an geistes- und literaturgeschichtlich bedeutsamen Werken und Formen, das von den indischen Veden über die Schriften kanonisierter Autoren bis hin zu zeitgenössischen Neuerscheinungen reicht: Die bereits 1918 begonnenen Notizen zur indischen Philosophie zeugen von seinen Schopenhauerinspirierten Lektüren der Upanischaden. Shakespeare und Goethe fanden ebenso

\footnotetext{
17 Vgl. Magdalena Gronau, Martin Gronau, »>mit saurem Schweiß sagen, was man nicht weiß<. Erwin Schrödinger und die Popularisierung der modernen Physik im Geiste der Anschauung «, Scientia Poetica 24 (2020), 213-253.

18 Vgl. Hans Ulrich Gumbrecht, »Das riskante Denken der Gegenwart. Es sind die Ingenieure und Computerspezialisten, die unsere Welt umgestalten, nicht die nach Wahrheit suchenden Naturwissenschaftler«, Neue Zürcher Zeitung (17.11.2016).

19 Detlef Dürr, Schrödingers Katze oder Wie kommt der Irrsinn zustande? Weihnachtsvorlesung 2006/07, in: https://www.mathematik.uni-muenchen.de/ duerr/KatzeWS06-07.pdf (08.10.2021).

20 Brief von Wolfgang Pauli an Erwin Schrödinger vom 26.06.1952, zit. nach Meyenn (Anm. 4), hier: 663.

21 Vgl. Arne Schirrmacher, »Kosmos, Koralle und Kultur-Milieu. Zur Bedeutung der populären Wissenschaftsvermittlung im späten Kaiserreich und in der Weimarer Republik «, Berichte zur Wissenschaftsgeschichte 31 (2008), 353-371.
}

22 Vgl. Jakobs (Anm. 9). 
selbstverständlich Eingang in seine Briefe und populären Aufsätze wie Grillparzer oder Zola. Ähnlich wie der Goethe-Liebhaber Werner Heisenberg ${ }^{23}$ in seiner dialogisch strukturierten Autobiografie knüpfte Schrödinger in seinem nachgelassenen

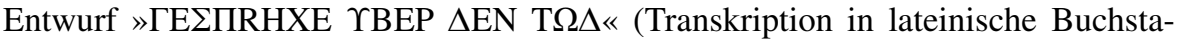
ben: »Gespräche über den Tod «) an die traditionsreiche literarische Form des antiken Lehrdialogs an. ${ }^{24}$ Kurz vor seinem Tod griff er das im 20. Jahrhundert gerade unter Naturwissenschaftlern florierende Genre der Autobiografie auf und schrieb einen essayistisch-philosophischen Lebensabriss. Und bereits in den 1940er-Jahren wies er sich auch öffentlich nicht nur als Literaturkenner, sondern als aktiver Poet aus, indem er ein Bändchen mit formal recht traditionellen Gedichten, zumeist Liebeslyrik, oft in Sonettform, veröffentlichte - übrigens im Verlag Helmut Küpper, der in der Nachfolge von Georg Bondi die Verlegung von Stefan Georges Werk weiterbetreute. ${ }^{25}$

\section{III.}

\section{Schrödinger und die (Kultur)Wissenschaftskultur der damaligen Zeit}

Schrödinger ist in seinem Rückgriff auf Literatur, wie bereits angedeutet, alles andere als ein Einzelfall. Es verwundert daher nicht, dass auch die modernen Literaturund Kulturwissenschaften die Entwicklung von Quanten- und Relativitätstheorie in den Blick genommen haben und sich für Korrelationen zwischen Wandlungen im Weltbild der Physik und zeitgenössischen literarisch-künstlerischen Innovationen interessieren. ${ }^{26}$ Etwas erstaunlicher ist schon der Umstand, dass sie damit, manchmal ohne es zu wissen, an allgemeine (wissenschafts)kulturanalytische Reflexionen der betreffenden Physiker anschließen. Zumeist essayistisch angelegt, sind solche Texte vor dem Hintergrund der jeweiligen individuellen Lebens- und Leseerfahrung $\mathrm{zu}$ verstehen, erreichten fallweise aber auch ein systematisches Erkenntnisniveau.

\footnotetext{
23 Skowronski (Anm. 10).

24 Schrödinger selbst hat, wohl versehentlich, bei seiner ungewöhnlichen Typisierung des deutschsprachigen Titels statt des griechischen $>\mathrm{P}<$ ein lateinisches $>\mathrm{R}<$ im Wort $\gg$ Gespräche« verwendet. Viele seiner Notizhefte sind mit griechischen Lettern versehen - im Unterschied zum hier beschriebenen Fall verwendete er aber für gewöhnlich altgriechische und keine deutschen Wörter.

25 Erwin Schrödinger, Gedichte, Godesberg 1949. Ganz ähnlich dichtete, um ein weiteres Beispiel aufzugreifen, der Haus- und Hofphilosoph der deutschsprachigen Quantenphysik Carl Friedrich von Weizsäcker.

26 Vgl. bspw. Sören Stange, »Vor-Augen-Entstellen«, Internationales Archiv für Sozialgeschichte der deutschen Literatur 39 (2014), 46-71; Sören Stange, Unentscheidbarkeiten. Zum Nicht-Wissen in Literatur und Naturwissenschaft um 1928, Paderborn 2014; Dirk Vanderbeke, Worüber man nicht sprechen kann. Aspekte der Undarstellbarkeit in Philosophie, Naturwissenschaft und Literatur, Stuttgart 1995; Dirk Vanderbeke, Theoretische Welten und literarische Transformationen. Die Naturwissenschaften im Spiegel der >science studies und der englischen Literatur des ausgehenden 20. Jahrhunderts, Tübingen 2004; Dirk Vanderbeke, »Überlegungen zur Ästhetik in der Literatur und in den Naturwissenschaften«, in: Aura Maria Heydenreich, Klaus Mecke (Hrsg.), Quarks and Letters. Naturwissenschaften in der Literatur und Kultur der Gegenwart, Berlin, Boston 2015, 117-140; Serpil Oppermann, »Quantum Physics and Literature. How They Meet the Universe Halfway«, Anglia 133 (2015), 87-104; Christoph Lehner, Jürgen Renn, Matthias Schemmel (Hrsg.), Einstein and the Changing Worldviews of Physics, Dordrecht u.a. 2012.
} 
So hatte Erwin Schrödinger in allgemein verständlichen Aufsätzen schon früh eine Interpenetration verschiedener gesellschaftlicher Sphären postuliert, besonders prominent in seinem 1932 in Schriftform veröffentlichten Vortrag »Ist die Naturwissenschaft milieubedingt? «. ${ }^{27}$ Ausgehend von der Beobachtung, dass der Physiker nicht nur als Forscher in seinem universitären Elfenbeinturm, sondern auch als Romane lesendes, Musik oder Kunst rezipierendes, Politik treibendes, gesellschaftlich aktives »Mitglied[] eines Kulturmilieus« agiere und als solches seine wissenschaftlichen Forschungen unter spezifischen, kulturell bedingten Interessenskonstellationen betreibe, diagnostizierte Schrödinger eine milieuspezifische Formatierung wissenschaftlicher Arbeit. Es bedürfe »eine[r] ganz spezielle[n] Interesseneinstellung [...], um von den vielen möglichen Fragen, die man an die Natur stellen kann, einige für augenblicklich sehr belangreich und wichtig, andere für belanglos zu halten. $\ll^{28}$ Diese These an einigen Beispielen exemplifizierend, verschaltet der Aufsatz etwa die bürokratische Verwaltung der Massen samt ihren Praktiken der Registratur, Erfassung etc. mit dem Aufschwung der Statistik in der Physik oder verbindet unter dem Stichwort »reine Sachlichkeit « gefasste Tendenzen in der modernen Kunst und Architektur, die sich u.a. durch ihren Mut zur ornamentlosen, leeren Fläche auszeichnen, mit einem schwindenden »horror vacui« der modernen Physik und der zunehmenden Akzeptanz prinzipieller, erkenntnistheoretisch fundierter Leerstellen im Wissen über die Natur.

Eine solche Verknüpfung von Ästhetik, Gesellschaft und Wissenschaft, heute, wenn man will, lesbar als eine intuitive Form der Wissenspoetologie avant la lettre, wirkt bei einem Physiker der damaligen Zeit zunächst einmal ausgesprochen modern. Jedoch darf nicht vergessen werden, dass Schrödingers Überlegungen zur Milieubedingtheit der Naturwissenschaft oder auch Cassirers bis 1936 abgeschlossene Studie zum (In)Determinismus der modernen Physik letztlich nur zwei von vielen Versuchen im frühen 20. Jahrhundert waren, die bahnbrechenden Erkenntnisse der neuen Physik wissenschaftstheoretisch zu systematisieren, zu historisieren und zu kulturalisieren. ${ }^{29}$ Es waren nicht selten >Naturforscher $<$ selbst, wie die zum Standardrepertoire der heutigen Wissenschaftsgeschichte avancierten Wissenschaftstheoretiker Ludwik Fleck oder Gaston Bachelard, die sich aus verschiedenen Perspektiven um eine adäquate Verortung der Forschungen ihrer Zeit bemühten - und die allgemein verständlichen Schriften der Physiker ebenso studierten ${ }^{30}$ wie umgekehrt etwa

27 Erwin Schrödinger, Über Indeterminismus in der Physik - Ist die Naturwissenschaft milieubedingt. Zwei Vorträge zur Kritik der naturwissenschaftlichen Erkenntnis, Leipzig 1932.

28 Schrödinger (Anm. 27), hier: 33.

29 Vgl. dazu die Ausführungen von Hans-Jörg Rheinberger, »Heidegger und Cassirer über die Wissenschaften nach der Begegnung in Davos«, Deutsche Zeitschrift für Philosophie 64 (2016), 655-666, der auf entsprechende Tendenzen in Philosophie und Kulturgeschichte der damaligen Zeit hinweist.

30 »Wie Bachelard verfolgte Fleck die von Niels Bohr, Werner Heisenberg und Erwin Schrödinger im allgemein-philosophischen Teil der Zeitschrift Die Naturwissenschaften geführten Debatten und verallgemeinerte die von Bohr auf das Quantenpostulat bezogene Relativierung des Erkenntnisprojektes epistemologisch.« Ernst Müller, Falko Schmieder, Begriffsgeschichte und historische Semantik. Ein kritisches Kompendium, Berlin 2016, 538. Auch Flecks Schrödinger-Lektüre ist durch seine Kritik an »Ist die Naturwissenschaft milieubedingt« verbrieft, vgl. dazu Claus Zittel, »Ludwik Fleck und der Stilbegriff in den Naturwissenschaften. Stil als wissenschaftshistorische, epistemologische und ästhetische Kategorie«, in: Horst Bredekamp, John Krois (Hrsg.), Sehen und Handeln, Berlin 2011, 171-206, hier: 197-198. 
Schrödinger schon als junger Forscher Wissenschaftsphilosophen wie Pierre Duhem rezipierte und sogar rezensierte. ${ }^{31}$ Schrödingers Brief an Cassirer jedenfalls lässt bereits im Jahr 1937 deutlich den Überdruss des Physikers an den Debatten um seine Disziplin erkennen: »Es mag an einer Übersättigung mit - fremden und eigenen die prinzipiellen Fragen meiner Wissenschaft betreffenden Gedanken liegen, einer Übersättigung, die durch das schmerzliche Bewusstsein, bei alledem doch nicht satt geworden zu sein, sich langsam zum Widerwillen gegen weitere Nahrungsaufnahme steigert. $\ll^{32}$

Solcher Abneigung gegenüber zeitgenössischer Physiker-Philosophie und Philosophen-Physik zum Trotz lag Schrödinger mit seinem Vortrag zur Milieubedingtheit seiner Disziplin durchaus im Trend seiner Zeit. Der Text fügt sich in die von HansJörg Rheinberger konstatierte Konjunktur epistemologischer Reflexionen im frühen 20. Jahrhundert ${ }^{33}$ - auch wenn Schrödinger dem gewählten Genre entsprechend eher offene Thesen in den Raum stellte als sie im Detail zu belegen, auch wenn er ohne Anspruch auf methodische Fundierung argumentierte und die neueren Tendenzen seiner Disziplin anders als etwa Fleck weniger mit einem »historischen Index « (Rheinberger) versah als mit einem sozio-kulturellen. Es ist bezeichnend für die Diversität der zeitgenössischen Debatte um die (Natur)Wissenschaftskultur, dass neben Fachgenossen aus der Physik auch Ludwik Fleck durchaus kritisch auf Schrödingers Überlegungen reagierte und die von Letzterem postulierten stilistischen Verwandtschaftsbezüge zwischen verschiedenen Gesellschaftsbereichen infrage stellte: »ein solcher - eher künstlerischer und literarischer als wissenschaftlicher Ansatz, [...] der auf einem intuitiven Erfühlen von Gemeinsamkeiten [...] und Zusammenhängen beruht [...], eignet sich noch nicht für die Forschung. Zu viel Literarisches und Beliebiges ist darin: Aus einem schönen Text herausgerissene Sätze überzeugen, kalt betrachtet, niemanden. Der Forschungsgegenstand löst sich auf, verschwindet wie ein spiritistisches Trugbild im Tageslicht. $\ll^{34}$

$\mathrm{Zu}$ beachten ist indes, dass sich Flecks Kritik an Schrödinger generell gegen einen seit der Jahrhundertwende ubiquitär gewordenen Stilbegriff richtet, der ursprünglich kunstgeschichtlich konnotiert war, nun aber auch in der Epistemologie Anwendung fand. In Abgrenzung davon bezieht sich der Fleck'sche Begriff des >Denkstils< sehr präzise und eng auf einzelne (Sub-)Disziplinen und ihre jeweiligen >Denkkollektive<, die weniger mit Blick auf vage Berührungspunkte und Gemeinsamkeiten mit anderen Bereichen des menschlichen Lebens denn in ihren konkreten, fachspezifischen Besonderheiten und Differenzen zueinander erfasst werden sollen. Unabhängig da-

\footnotetext{
${ }^{31}$ Erwin Schrödinger, »Die Wandlungen der Mechanik und die mechanische Naturerklärung. Von P. Duhem. (L'evolution de la mecanique). Übersetzt von Philipp Frank. Leipzig 1912«, Monatshefte für Mathematik und Physik 24 (1913), 25-27. Diese Auseinandersetzung Schrödingers mit Duhem scheint den Wissenschaftshistoriker/inne/n, welche sich die Sammlung von Schrödingers Gesamtwerk in den 1980erJahren zur Aufgabe gemacht hatten, entgangen zu sein. Die Rezension scheint jedenfalls nicht in den $\mathrm{Ge}$ sammelten Abhandlungen auf; auch eine wissenschaftshistorische Aufarbeitung des Sachverhalts ist uns nicht bekannt.

32 Schrödinger an Cassirer (Anm. 4).

${ }^{33}$ Rheinberger (Anm. 29), hier: 666.

${ }^{34}$ Ludwik Fleck, Denkstile und Tatsachen. Gesammelte Schriften und Zeugnisse, hrsg. Sylwia Werner, Claus Zittel, Frankfurt a.M. 2011, 330.
} 
von, ob sie berechtigt ist, liefert Flecks Kritik einen Ansatzpunkt zur Präzisierung der hier aufgeworfenen Frage nach historischen Entwicklungen im Verhältnis der modernen Physik zur Literatur. Denn wenn die Feststellung von positiven Bezügen der Naturwissenschaftler auf Literatur und entsprechenden Affinitäten zwischen Physik, Literatur und Kunst heutzutage fast schon als Gemeinplatz zu bezeichnen ist, der bereits zur damaligen Zeit von Forschern diverser fachlicher Provenienz zum Gegenstand jeweils unterschiedlich perspektivierter Reflexionen gemacht wurde, bedarf es womöglich einer Verengung des analytischen Fokus. Eine erste Möglichkeit wäre zu fragen, ob Schrödinger (ebenso wie Cassirer) eigentlich >die< Physik an sich bzw. den Physiker im Allgemeinen im Blick hatte, oder aber einen spezifisch historisierbaren Typus, nämlich den theoretisch arbeitenden Physiker, dessen alltägliche Arbeitspraxis völlig andere mediale und technische Bedingungen erforderte als die auf das Labor und darin durchgeführte Operationen konzentrierte Tätigkeit des Experimentalphysikers. Lassen sich in der noch kleinen Gemeinschaft der theoretischen Physik, die sich mit den bahnbrechenden Erkenntnissen Plancks und Einsteins als institutionell eigenständige Subdisziplin etablieren konnte und sich seither - gerade im deutschen Sprachraum - zunehmend von der Experimentalphysik abgrenzte, ${ }^{35}$ Eigenheiten im Verhältnis zu Literatur, Sprache und damit verbundenen Wissensformationen feststellen? Und sind diese Besonderheiten, um Schrödinger und Fleck gewissermaßen zur Synthese zu bringen, möglicherweise charakteristisch für den >Denkstil< der modernen theoretischen Physik?

\section{IV.}

\section{Schrödinger und die Philologie}

Innerhalb der theoretischen Physik wurde die Entwicklung der Quantentheorie von ausgedehnten Debatten um eine adäquate Beschreibung intuitiv nicht zugänglicher Phänomene begleitet: Zwar konnte die Quantenphysik mathematisch präzise erfasst werden, doch scheiterten die Physiker an der Übersetzung der Formalismen in die Alltagssprache mit ihren auf Anschauung und Sinneserfahrung fußenden klassischen Begrifflichkeiten. Von der intensiven Auseinandersetzung mit dem problematischen Verhältnis von Sprache und Naturerkenntnis zeugen neben themenspezifischen Auf-

\footnotetext{
35 Ausführliche Erläuterungen zur Entwicklung der theoretischen Physik in Deutschland finden sich in Eckert (Anm. 5).
} 
sätzen der Physiker auch Briefe und retrospektive Reflexionen, ${ }^{36}$ welche eine Vielfalt unterschiedlicher Positionen in Detailfragen und damit verbundene Grabenkämpfe innerhalb der jungen Subdisziplin sichtbar machen. Werner Heisenberg etwa kolportiert in seiner Autobiografie die Anekdote, dass Erwin Schrödinger, während eines Gastaufenthalts in Kopenhagen erkrankt und von Niels Bohrs Frau gepflegt, die Diskussionen um die Möglichkeit einer anschaulichen Interpretation der Quantenmechanik mit dem dänischen Kollegen noch im Krankenbett fortsetzen musste. ${ }^{37}$ In einem Brief an den Kollegen und Freund Max Born beschließt Schrödinger wiederum süffisant seine Ausführungen zu einer Bohr'schen Passage, in der dieser die notwendige, dabei jedoch, so zumindest Bohr, stets ungenügende Beschreibung jeglicher Beobachtung in »common language « hervorhebt: »Der eminente Physiker Niels Bohr wird als >Philosopher-Scientist< von Seiten seiner Physikerkollegen eminent überschätzt. Das [Bohrs Überschätzung, MMG] ist ein sehr gewöhnlicher Sachverhalt, der sich in gewöhnlicher Umgangssprache sehr leicht beschreiben und begründen läßt. $\ll^{38}$

Die atomphysikalischen Rekurse auf Sprache sind in ihrer Ausprägung und Funktion überaus vielschichtig; sie beschränken sich keineswegs auf zeittypische Reflexionen über die >Grenzen der Sprache sowie damit verbundene epistemologische Ansätze einer quantentheoretisch fundierten $>($ Sprach-)Philosophie der Physiker $<: 39$ Ein mikroskopischer Blick in physikalische Fachschriften offenbart eine fast schon symptomatische Häufung von sprachbezogenen Genitivattributen wie »Sprache der Wellen«, »Sprache der Teilchen«, »Sprache der Aethertheorie «, »Sprache der bisherigen Theorie «, »Sprache der Elektronenbahnentheorie « etc. ${ }^{40}$ Das Konzept der >Sprache< mit ihrem Wortschatz, ihrem grammatikalischen Regelwerk und insbesondere dem darin eingeschlossenen Translationspotenzial bildete für die Quantenphysiker offenbar einen wichtigen Referenzbereich, um sich über die Erstellung und Übertragung verschiedener (mathematischer) Ansätze zur Beschreibung physikali-

\footnotetext{
36 Siehe z.B. Werner Heisenberg, »Sprache und Wirklichkeit in der modernen Physik «, in: Wort und Wirklichkeit. Sechste Folge des Jahrbuches >Gestalt und Gedanke`, München 1960, 32-62. Vgl. dazu auch Peter Mittelstaedt, »Die Sprache der Physik«, in: Hans-Peter Dürr (Hrsg.), Quanten und Felder. Physikalische und philosophische Betrachtungen zum 70. Geburtstag von Werner Heisenberg, Braunschweig 1971, 27-50; Peter Mittelstaedt, Sprache und Realität in der modernen Physik, Mannheim 1986; Brigitte Falkenburg, »Sprache und Anschauung in der modernen Physik«, in: Herbert Ernst Wiegand (Hrsg.), Sprache und Sprachen in den Wissenschaften. Geschichte und Gegenwart, Festschrift für Walter de Gruyter \& Co. anlässlich einer 250-jährigen Verlagstradition, Berlin, New York 1999, 89-118; Matthias Dörries, »Modern Science and the Spirit of Language, Literature and Philology«, in: Miles MacLeod, Rocío G. Sumillera, Jan Surman, Ekaterina Smirnova (Hrsg.), Language as a Scientific Tool. Shaping Scientific Language Across Time and National Tradition, London, New York 2016, 9-21.

37 Werner Heisenberg, Der Teil und das Ganze. Gespräche im Umkreis der Atomphysik, München 1969, 108-109.

38 Brief von Erwin Schrödinger an Max Born vom 28.06.1952, zit. nach Meyenn (Anm. 4), hier: 665.

39 Erhard Scheibe, Die Philosophie der Physiker, München 2006.

40 Beispiele finden sich in Erwin Schrödinger, »Quantisierung als Eigenwertproblem. Zweite Mitteilung«, Annalen der Physik 79 (1926), 489-527, hier: 526; Erwin Schrödinger, »Quantisierung als Eigenwertproblem. Dritte Mitteilung«, Annalen der Physik 80 (1926), 437-490, hier: 439, 475. Erwin Schrödinger, »Quantisierung als Eigenwertproblem. Vierte Mitteilung«, Annalen der Physik 81 (1926), 109-139, hier: 122 .
} 
scher Phänomene zu verständigen, die dem sprachlich beschränkten Alltagsverstand, dem gemeinen Logos, nicht mehr zugänglich waren. ${ }^{41}$

Interessant ist vor allem, dass jenseits solch metaphorischer Konzeptionen auch natürliche Sprachen an sich eine nicht unerhebliche Rolle im Werk der Atomphysiker spielen - und zwar nicht nur in beiläufigen »Versuch[en] einer vernünftigen Schreibung des Englischen $\ll,{ }^{42}$ wie sie sich beispielsweise im Nachlass Schrödingers finden. Während nach Ansicht mancher Wissenschaftshistoriker die Mathematik im Begriff war, sich als >Muttersprache der durchrationalisierten Moderne ${ }^{43} \mathrm{zu}$ etablieren, kultivierte ein Großteil der theoretischen Physiker, deren Kerngeschäft gerade in jener Mathematisierung bestand, auch schon vor etwaigen Exilerfahrungen eine grundständige Form der Polyglottie. Diese war bemerkenswerterweise keineswegs nur dem lebendigen internationalen Wissensaustausch dienlich, sondern zeichnete sich durch eine auffällige Affinität zu den Toten bzw. Alten Sprachen aus, insbesondere zum Griechischen. Im Fall Schrödingers tritt diese Neigung etwa in wissenschaftshistorischen Versuchen mit Titeln wie »2400 Jahre Quantentheorie «44 zutage, in denen er gängige Interpretationen der Quantenmechanik mit der griechischen Atomistik verschaltete und mitunter sogar vorsokratische Fragmente in Originalsprache zitierte. Auch die im Nachlass erhaltenen »griechischen Präparationen« aus Schrödingers Wiener Gymnasialzeit, in denen sich u.a. seine Mitschriften zur griechischen Naturphilosophie finden, lassen auf den hohen Stellenwert schließen, den der Physiker dem Altgriechischen zuschrieb. Besonders eindrücklich zeugen Schrödingers Annotationen in thematisch einschlägigen Fremdseparata ${ }^{45}$ sowie seine Angewohnheit, Notizbücher mit griechischen Titeln bzw. Lettern zu versehen, von seinem anhaltenden Philhellenismus.

Der im Brief an Cassirer behaupteten Abneigung des »echten Naturforscher[s] von heute « gegen überkommene philosophische Begriffsschemata zum Trotz beschäftigte sich Schrödinger also selbst in ähnlich intensiver Weise wie Werner Heisenberg, Wolfgang Pauli oder Carl Friedrich von Weizsäcker mit der Naturphilosophie und Sprache des Altertums. Und auch wenn die theoretische Physik der damaligen Zeit mit Blick auf die jeweils bevorzugten philosophisch-literarischen »Hexen-

\footnotetext{
41 Beispielsweise betonte Werner Heisenberg in einem Brief an Wolfgang Pauli vom 24.06.1925, also in jenem Jahr, in dem ihm gemeinsam mit Max Born und Pascual Jordan der Durchbruch in der Entwicklung der Matrizenmechanik gelang, die Bedeutung der »Voss'schen Methode« als Schlüssel zur >Übertragung « klassisch-physikalischer Beschreibungsansätze in die neuartigen quantenmechanischen. Vgl. hierzu Alexander Blum, Martin Jähnert, Christoph Lehner, Jürgen Renn, »Translation as heuristics. Heisenberg's turn to matrix mechanics«, Studies in History and Philosophy of Modern Physics 60 (2017), 3-22, hier: 16.

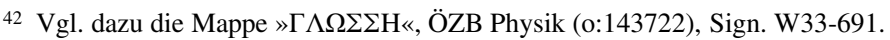

43 Vgl. Herbert Mehrtens, Moderne - Sprache - Mathematik. Eine Geschichte des Streits um die Grundlagen der Disziplin und des Subjekts formaler Systeme, Frankfurt a.M. 1990.

44 Erwin Schrödinger, »2400 Jahre Quantentheorie«, Annalen der Physik 6/3 (1948), 43-48.

45 Vgl. z.B. eine philhellenische Marginalie in einem Schrödinger zugesandten Aufsatz von Max Hartmann mit dem Titel »Naturwissenschaft und humanistische Bildung«, in dem von der »logischen Klarheit der alten Sprachen« die Rede ist. Dort hat Schrödinger vermerkt: »Plural? Einer davon! Und zwar nicht der lateinischen!« Der Aufsatz ist im Nachlass Schrödingers in einem archivalisch nicht im Detail erfassten Fremdseparata-Konvolut an der ÖZB für Physik (Nachlass Schrödinger, Kasten 21) verwahrt.
} 
küchen « durchaus verschiedene Geschmäcker ausprägte, ${ }^{46}$ hatte Schrödinger wohl recht, wenn er seine Faszination von der griechischen Antike und ihrer Sprache in seinen öffentlichen Vorträgen $\mathrm{zu} »$ Nature and the Greeks« innerhalb eines »trend of thought rooted somehow in the intellectual situation of our time « verortete. ${ }^{47}$ Ähnlich wie seine Beobachtungen zur soziokulturellen Bedingtheit naturwissenschaftlicher Forschungstätigkeit zeugt diese Einschätzung vom feinen Sensorium des Physikers für das eigene Sozialmilieu und die zeitgenössische Wissenschaftskultur: Im Werk der deutschsprachigen Gründerväter der modernen Atomphysik lassen sich in der Tat erstaunlich dichte Spuren ihrer Affinität nicht nur zu Literatur, Sprache und Text, sondern auch und vor allem zur Textwissenschaft, also zur (klassischen) Philologie fassen. Im Folgenden wollen wir, wiederum am Beispiel Schrödingers, zeigen, auf welche Weise die theoretischen Physiker jener Zeit philologische Wissensformationen für ihre Forschungs- und Publikationspraxis fruchtbar machten. ${ }^{48}$

In einem ersten Schritt gehen wir der Frage nach, wie die Physiker dem Anschauungs- und Versprachlichungsproblem der modernen Quantenphysik mittels ihres humanistischen Bildungsreservoirs zu Leibe rückten. Ernst Cassirer hat in seinen wissenschaftsphilosophischen Schriften wiederholt betont, dass im Zuge der Entwicklung von Relativitäts- und Quantentheorie eine »Physik der Bilder und Modelle« von einer »Physik der Prinzipien « abgelöst worden sei. ${ }^{49}$ Der darin eingeschlossenen epistemologischen Ambivalenzen war sich der Philosoph bewusst: In der auch Schrödinger zugesandten Schrift Determinismus und Indeterminismus in der modernen Physik verwies er mit Blick auf Du Bois Reymond, der ja mit seinem »Ignoramus et Ignorabimus « im frühen 20. Jahrhundert intensiv rezipiert wurde, auf die »Hypostase « der grundlegenden Erkenntnisinstrumente »Sprache und Bild «. »In dieser Hypostase « werde »das Instrument «, im Fall der theoretischen Physik also der mathematische Formalismus, »zu einer selbständigen, eigentümlichen und eigenwilligen Kraft, die den Menschen in ihren Bann zieht. [...] Das Symbolische wird zum Magischen«. Und weiter: »So merkwürdig und paradox es scheinen mag, so ist doch selbst die >abstrakteste< Symbolbildung von diesem Zwange zum unmittelbar Bildhaften, und damit von dem Zwange zur Verdinglichung, nicht frei. $\ll^{50}$ Hier lassen sich durchaus Verbindungen zu Schrödingers Positionen in der aufgeladenen Anschaulichkeitsdebatte der Quantenphysik ziehen: Der vielseitige Theoretiker wehrte sich schließlich zeit seines Lebens gegen den >Bildersturm< seiner Fachgenossen, die das Beharren auf der Möglichkeit einer »anschaulichen« Beschreibung quan-

\footnotetext{
46 Während Werner Heisenberg oder Carl Friedrich von Weizsäcker sich gerne als idealistische Platoniker inszenierten, bezog Schrödinger seine Inspirationen vornehmlich aus vorsokratischen Quellen und distanzierte sich ausdrücklich vom »unglücklichen Erbe[] der Nachsokratiker«. Brief von Erwin Schrödinger an Arnold Sommerfeld (13.02.1949), zit. nach Meyenn (Anm. 4), hier: 621. Vgl. auch Hellmut Flashar, »Zettel's Traum. Georg Picht und das Platon-Archiv in Hinterzarten«, Zeitschrift für Ideengeschichte 5/1 (2011), 94-104.

47 Erwin Schrödinger, Nature and the Greeks and Science and Humanism, Canto Classics, Cambridge 2014, 4.

48 Vgl. dazu auch Georg Toepfer, Hartmut Böhme (Hrsg.), Transformationen antiker Wissenschaften, Berlin, Boston 2010.

49 Cassirer (Anm. 1), hier: 13:533.

50 Cassirer (Anm. 1), hier: 19:15.
} 
tenphysikalischer Phänomene vereinzelt als regressive Sehnsucht nach den alten Vertrautheiten der klassischen Physik abtaten. ${ }^{51}$ Schrödingers an mehreren Stellen explizit formulierte Epistemologie der Anschauung blieb nicht nur graue Theorie, sondern fand ihren Niederschlag auch in seiner anschauungszentrierten Forschungsund Darstellungspraxis. So benutzte er mit Vorliebe Bilder, auch sprachlicher Natur - Metaphern, Vergleiche etc. -, um schwierige wissenschaftliche Tatsachen dem interessierten Laien, aber auch dem Fachmann verständlich zu machen. ${ }^{52}$ Gerade die griechische Antike bot ihm ein reichhaltiges Reservoir an Inspirationen: Mythischsurreale Fabelwesen eigneten sich beispielsweise hervorragend, um alogische quantenmechanische Phänomene zu versinnbildlichen und damit dem Alltagsverstand zugänglicher zu machen. ${ }^{53}$ In Einzelfällen ließ sich - so z.B. im Fall einer gekritzelten Chimäre, die als Sinnbild des >unmöglichen< Welle-Teilchen-Dualismus Bohrs Komplementaritätsprinzip ins Lächerliche zieht - mit derartigen Übertragungen literarischen Kanonwissens noch eine Kritik an der quasi-religiösen Dogmatik verbinden, mit der, zumindest aus Schrödingers Perspektive, Bohrs Anhänger die Kopenhagener Deutung zum Glaubenssatz hypostasierten, der mathematischen Formulierung den Primat einräumten und allein schon die Möglichkeit einer anschaulichen Interpretation in Abrede stellten. ${ }^{54}$

Unabhängig von erkenntnistheoretischen Divergenzen ließen sich neben Schrödinger auch andere Physiker von philologischen Inhalten inspirieren. Werner Heisenberg, um nur ein Beispiel zu nennen, war vor allem nachsokratischen Inspirationsquellen zugänglich und stilisierte in der autobiografischen Rückschau seine frühmorgendlichen Timaios-Lektüren zum atomphysikalischen Erweckungserlebnis. ${ }^{55}$ Inhaltlich-konzeptuell griff er immer wieder auf Platon oder Aristoteles zurück und sprach beispielsweise bei der Beschreibung der Wellenfunktion $\psi$ und der daraus ableitbaren Aufenthaltswahrscheinlichkeit des Elektrons von einer »quantitativen Fassung des Begriffs der $\delta v ́ v \alpha \mu \iota$, , [...] der >potentia< in der Philosophie des Aristoteles. ${ }^{56}$ Ähnlich funktionalisierte Verweise auf altgriechische Begriffswelten - diesmal jedoch auf vorsokratische und insbesondere Heraklitische - finden sich auch bei Schrödinger, in wissenschaftshistorischen bzw. -philosophischen Aufsätzen ebenso wie im schriftlichen Austausch mit Kollegen. ${ }^{57}$ In einem Brief aus dem Jahr 1952 an Max Born etwa setzte Schrödinger sich mit einem ihm zugesand-

51 Vgl. z.B. den Brief von Wolfgang Pauli an Erwin Schrödinger (26.06.1952), zit. nach Meyenn (Anm. 4), hier: 663 .

52 Vgl. etwa seine Notizen zur »Begrenzung der Naturwissenschaft«, in ÖZB Physik (o:143722), Sign. W33-315. Weiterführende Informationen und Analysen zu Schrödingers auf Anschaulichkeit abgestelltem Theorie-Begriff in Gronau, Gronau (Anm. 17), hier: v.a. 244-251.

$53 \mathrm{Vgl}$. dazu einen für mit der Quantentheorie nicht vertrauten Kollegen geschriebenen Aufsatz, in dem wiederholt von einer »Zentaurennatur« der Welt die Rede ist. Erwin Schrödinger, »Neue Wege in der Physik«, Elektrotechnische Physik 50 (1929), 15-16.

54 Vgl. Meyenn (Anm. 4), hier: 573.

55 Heisenberg (Anm. 37), hier: 20.

56 Vgl. dazu Werner Heisenberg, »Die Plancksche Entdeckung und die philosophischen Probleme der Atomphysik «, Universitas 14 (1959), 135-148, hier: 140.

57 Beispielsweise in Erwin Schrödinger, »Die Besonderheit des Weltbilds der Naturwissenschaft«, in: Was ist ein Naturgesetz? Beiträge zum naturwissenschaftlichen Weltbild, München 1997, 27-85. 
ten Manuskript zu einem Vortrag auseinander, den der Freund einige Monate früher in London zum Problem der Physical Reality gehalten hatte: ${ }^{58}$

p. 20: »It has under certain circumstances not a distinct individuality. «

To this I say

Gibt's etwas [sic!] da ein Weniger oder Mehr?

In any circumstances whatsoever I have to use - im Prinzip - the antisymmetric wave function.

Summary: Trotz meiner vielen Einwände bin ich mit dem Grundgedanken sehr einverstanden. Wenn Du's zur Hand hast, lies einmal in der ebengenannten Abhandlung (Besonderheit des Weltbildes ... ) meine Heraklit-Interpretation (p. 225) nach. Philologisch mag sie unzutreffend sein, aber dem Sinn nach deckt

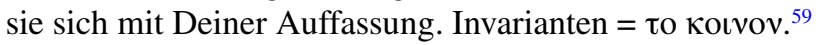

Diese merkwürdig zusammengezimmert wirkende Passage ist typisch für den Kommentarstil des lesenden Kritikers Schrödinger: Seine schriftlichen Diskussionsbeiträge schließen jeweils an einzelne, durch Seitenverweise näher bestimmte, besprechungswürdige Zitate aus dem Text des Kollegen an. Zugleich bündelt der versatzstückhafte Abschnitt exemplarisch die bisher andiskutierten - unspezifischen wie spezifischeren - Aspekte des Wechselverhältnisses zwischen Naturwissenschaft und Literatur, (theoretischer) Physik und Philologie. So zeichnet sich die Passage zunächst durch ein recht selbstverständliches Oszillieren zwischen der Muttersprache und der Sprache des Exillandes aus. Dabei handelt es sich freilich nicht um ein subdisziplinäres Charakteristikum: Die Sprachkompetenz Schrödingers, der schon als kleines Kind im Englischen geschult wurde ${ }^{60}$ und neben Latein und Altgriechisch auch Französisch, Spanisch und Italienisch zumindest passiv ausgezeichnet beherrschte, mitunter sogar Aufsätze in den jeweiligen Sprachen schrieb bzw. Selbstübersetzungen anfertigte, stellt lediglich einen Extremfall bildungsbürgerlicher Multilingualität dar. Ebenso typisch für die gelehrte Kommunikation jener Zeit sind literarhistorische Rekurse, mit denen man oft und gerne seine Korrespondenzen würzte - im konkreten Fall mit einer (wenn auch etwas schief, weil vermutlich aus dem Gedächtnis) zitierten Schillerzeile aus dem »Verschleierten Bildnis zu Sais «. ${ }^{61}$ Während diese Lesefrucht kaum inhaltliche Funktionen hat, sondern eher das Kanonwissen des gelehrten Briefeschreibers vorführt, ist der Verweis auf Heraklit bzw. Schrödingers eigene Heraklit-Auslegung semantisch vielschichtiger - und bezeichnend für

\footnotetext{
58 Aus dem Briefwechsel der Physiker wird ersichtlich, dass die beiden einen intensiven Austausch von Manuskripten bzw. Sonderdrucken pflegten, welche sie nicht nur von Angesicht zu Angesicht, sondern auch schriftlich diskutierten. Der hier in Rede stehende Vortrag wurde 1953 publiziert.

59 Schrödinger an Born (Anm. 37), hier: 666. Hier fehlende griechische Akzente, die Schrödinger in seinen handschriftlichen Griechisch-Notizen in den meisten Fällen korrekt anbringt, sind wahrscheinlich auf Auslassungen bei der Edierung der Originalbriefe zurückzuführen.

60 Schrödingers Großmutter mütterlicherseits war Britin; eine Tante, mit der er in Kindertagen viel Kontakt hatte, kommunizierte auf Englisch mit ihm. Einer überlieferten Anekdote zufolge beherrschte Schrödinger das Englische, noch bevor er vernünftig Deutsch sprechen konnte. Vgl. Walter J. Moore, Erwin Schrödinger. Eine Biographie, Darmstadt 2015, hier: 25.

61 In das Schiller-Zitat hat sich vermutlich ein Transkriptionsfehler in Form eines überschüssigen $>$ s $<$ eingeschlichen (»etwas« statt »etwa«).
} 
den Umgang der theoretischen Physiker jener Zeit mit philologischen Wissensformationen. Es handelt sich nicht um ein argumentum ad verecundiam im engeren Sinn, vielmehr bedient sich Schrödinger hier eines aus der frühesten Philosophiegeschichte überlieferten Konzepts, welches die von ihm behauptete »Überlappung « der individuellen Wirklichkeiten, i.e. die intersubjektive Realität, vermeintlich präziser zu fassen vermag als eine Beschreibung »in common language «, sei es nun in deutscher oder englischer. Gleichwohl ist die formelhaft mit einem Ist-gleich-Zeichen suggerierte Passgenauigkeit des Heraklitischen Konzepts weniger auf tatsächliche begriffliche Präzision denn vielmehr auf systematische Vagheit und Ungenauigkeit zurückzuführen: ${ }^{62}$ Wie Schrödingers Hinweis auf die philologische Fragwürdigkeit seiner »Interpretationen « andeutet, führt nicht zuletzt die im Fall der Vorsokratiker sehr zweifelhafte Quellenlage dazu, dass seine Auslegung eben nur eine von mehreren möglichen ist - allen philologischen Geschicks zum Trotz, über das der Physiker als ehemaliger Klassenprimus und ausgezeichneter Altgriechisch-Schüler ${ }^{63}$ selbstverständlich verfügte. Das Gleichheitszeichen suggeriert Identität, wo Schrödinger sich de facto im interpretativen Spielraum bewegt. Die auf solche Weise zutage tretende Dissonanz zwischen vermeintlich exakter Mathematik und interpretierender Sprache, zwischen >harter $<$ Rechen- und >weicher $<$ philologischer Interpretationsarbeit, klingt interessanterweise bereits in einigen polemischen Passagen im Werk Friedrich Nietzsches an. Als »alte[r] Philologe[], der von der Bosheit nicht lassen« konnte, nutzte dieser nämlich zeitweise die Gelegenheit, seine Kollegen aus der Physik zu kritisieren, auf deren »schlechte Interpretations-Künste den Finger« zu legen - und en passant entsprechende disziplinäre Zuschreibungen auf den Kopf zu stellen: »aber jene >Gesetzmässigkeit der Natur<, von der ihr Physiker so stolz redet, wie als ob - - besteht nur Dank eurer Ausdeutung und schlechten >Philologie <, sie ist kein Thatbestand, kein >Text<, vielmehr nur eine naiv-humanitäre Zurechtmachung und Sinnverdrehung, mit der ihr den demokratischen Instinkten der modernen Seele sattsam entgegenkommt! «4

Was Schrödingers Liebäugelei mit der Philologie betrifft, lässt sich wiederum festhalten, dass gerade die Vagheit von bruchstuickhaft tradiertem Wissen in den von ihm hergestellten Transformationszusammenhängen kein epistemisches Manko darstellte - ganz im Gegenteil: Sie dürfte mit dafür verantwortlich sein, dass antike und insbesondere vorsokratische Motive und Konzeptionen eine gewisse Attraktivität für die Übertragung in quantenphysikalische (Kon-)Texte entfalten konnten. Deutungsoffene, semantisch ambivalente Fragmente ließen sich je nach Standpunkt zur Beschreibung mathematisch-physikalischer Entitäten oder für erkenntnistheoretische Überlegungen fruchtbar machen. Indes beschränkte sich das interdisziplinäre Transformationspotenzial der seit dem 19. Jahrhundert florierenden Philologien keineswegs auf philologisch erschlossene literarische Motive und philosophische Begriffsschemata. Auch die Operationen und Techniken, mithilfe derer philologisches

\footnotetext{
62 Vgl. auch Michael Bies, Michael Gamper (Hrsg.), Literatur und Nicht-Wissen. Historische Konstellation 1730-1930, Zürich 2012.

63 Vgl. dazu Gabriele Kerber, Auguste Dick, Wolfgang Kerber (Hrsg.), Dokumente, Materialien und Bilder zur 100. Wiederkehr des Geburtstages von Erwin Schrödinger, Wien 1987, hier: 21.

${ }^{64}$ Friedrich Nietzsche, Jenseits von Gut und Böse, 1886, zit. nach: eKGWB/JGB-22.
} 
Wissen gesammelt, organisiert, verwaltet und hervorgebracht wurde, erwiesen sich für die theoretische Physik des frühen 20. Jahrhunderts als anschlussfähig. Eine sehr eigenwillige Form von >Goethe-Rezeption< in der von Robert Musil erwähnten Schrödinger'schen »Plauderei«, die in der populärwissenschaftlichen Zeitschrift Die Koralle veröffentlicht worden war, ${ }^{65}$ ist dafür symptomatisch. In dem kurzen, auf den Kontext der Illustrierten abgestimmten Artikel veranschaulicht Schrödinger am Beispiel einer in einer Bibliothek aufgestellten Goethe-Ausgabe statistische Grundlagen. An der (berechenbaren) Verteilung der ursprünglich zusammen im Regal aufgestellten Gesamtausgabe nach zufälligem Herausnehmen und Wieder-Einstellen einzelner Bände führt er vor, dass auch der Zufall, der dem interessierten Laien der damaligen Zeit zumindest vom Hörensagen als Schreckgespenst des klassischen Physikers und seines Kausalitätsprinzips bekannt war, bestimmten Gesetzmäßigkeiten (eben der Statistik) unterliegt. »Goethe« muss hier also nicht als Spender geflügelter Worte, als Identifikationsfigur des universell gebildeten Naturforschers oder gar als Kronzeuge nationaler Exzeptionalität ${ }^{66}$ herhalten. »Goethe« steht hier vielmehr für ein qua Autorschaft als zusammengehörig markiertes Ensemble von Dingen (im Schrödinger'schen Bild: von Büchern), die durch das »Gesetz des Zufalls« innerhalb eines größeren Ensembles permutiert werden. Dieser Rekurs auf Goethe ist symptomatisch für die Philologie-Bezogenheit der (theoretischen!) Physiker im Umgang mit Literatur im ersten Drittel des 20. Jahrhunderts: Zur Illustration der physikalischen Gesetzmäßigkeiten greift Schrödinger eben nicht auf vom Material losgelöste, dem Bildungsbürgertum der damaligen Zeit vertraute geistige Inhalte zurück, sondern auf »Goethe « als >Objekt $<-$ und auf die Wissens(an)ordnung der Bibliothek, innerhalb derer dieses Objekt nach bestimmten Kriterien einsortiert wird (oder eben nicht). Damit ruft er weniger ein literarisches Register denn ein philologisch-textwissenschaftliches auf - samt der damit verknüpften Praktiken, wie sie insbesondere die klassische Editionsphilologie ein paar Dekaden vorher im Zuge der Erschließung, Verwaltung und Bearbeitung rasant anwachsender, dabei aber fragmentarischer und höchst unsicherer Quellenbestände ausgebildet hatte. ${ }^{67}$ Es dürfte jedenfalls kein Zufall sein, dass Schrödinger in seinem Aufsatz zur »Besonderheit des Weltbilds der Naturwissenschaft « auf die zeitliche Koinzidenz der »mühevolle[n], sichtende[n] Sammlung « der Vorsokratiker-Fragmente durch den berühmten Altphilologen Hermann Diels mit der »Lebenszeit Machs « hinweist. ${ }^{68}$ Denn ähnlich wie die Philologie war auch die klassische Physik, die im 19. Jahrhundert bereits als abgeschlossen galt, mit explodierenden Datenmengen, neuen Erkenntnissen und damit verbundenen Verunsicherungen konfrontiert. Diese trugen maßgeblich zu den wissenschaftlichen Revolutionen der Quanten- und Relativitätstheorie sowie zur universitären Etablierung der theoretischen Physik bei, die noch um 1900 den Status eines ungeliebten Sidecuts der Experimentalphysik innehatte. Nicht zuletzt

\footnotetext{
65 Schrödinger (Anm. 16).

$66 \mathrm{Zu}$ auf solche Weise funktionalisierten Goethe-Bezügen bei Heisenberg vgl. Skowronski (Anm. 10).

67 Zur Modellfunktion der Philologie vgl. Denis Thouard, Friedrich Vollhardt, Fosca Mariani Zini (Hrsg.), Philologie als Wissensmodell. La philologie comme modèle de savoir, Berlin, Boston 2010.

68 Erwin Schrödinger, »Die Besonderheit des Weltbilds der Naturwissenschaft«, Acta Physica Austriaca 1/3 (1948), 201-245, zit. nach Schrödinger (Anm. 57), hier: 29.
} 
machten sie sich jedoch auf praktischer Ebene bemerkbar: Im Umgang mit sich beständig vergrößernden, schwierig einsortierbaren, unklaren und interpretationsoffenen Wissensbeständen war die Entwicklung neuer Arbeitstechniken unumgänglich geworden.

Schrödingers didaktisches Bild von der Goethe-Ausgabe verweist bereits auf eine gewisse philologisch affizierte Praxis- und Materialbezogenheit der theoretischen Physik jener Zeit. Nichtsdestoweniger operiert es selbst, als anschauliches Gleichnis in einem populärwissenschaftlichen Aufsatz, das der Illustration von komplexen mathematischen Sachverhalten dient, auf der Ebene der Sprache. Die vielfältigen Ausprägungen der >Philologie der Physiker< geben hingegen Anlass zu der Vermutung, dass die von uns postulierte Affinität von theoretischer Physik und Philologie über eine eher intuitiv wahrnehmbare, ästhetisch-stilistische Verwandtschaftsbeziehung hinausgeht. Dafür spricht der Umstand, dass die Neigung der theoretischen Physiker zum Literarisch-Philologischen selbst in ihrer wissenschaftlichen Arbeitspraxis manifest werden konnte. Hinweise darauf finden sich etwa in Schrödingers Notizheften, die aufschlussreiche Titel wie » $\Lambda \Omega \Sigma \Sigma H \ll$ (Transkription: »Glosse«, übersetzt: »Zunge, Sprachfertigkeit, Sprache «) tragen, ${ }^{69}$ und neben literarischen Versuchen beispielsweise Notizen zum »Bedeutungswandel« von Wörtern oder zum Verhältnis von »Sprache und Begriffen« enthalten. Als besonders aufschlussreich erweist sich eine Mappe, die an der Zentralbibliothek für Physik der Universität Wien als Kopie verwahrt wird und im digitalen Nachlass-System PHAIDRA nicht erfasst ist. In dem Heft, das mit dem Wort »POIH $\Sigma \mathrm{I} \Sigma$ « (Transkription: »Poiesis«) übertitelt ist, finden sich viele Gedichte, datiert auf das Jahr 1928 und damit auf die Hochphase von Schrödingers wissenschaftlicher Produktivität. Das zeigt zunächst einmal, dass Schrödingers literarische Ambitionen nicht nur dem gängigen Format der gelehrten Alterslyrik zuzuordnen sind, dessen sich viele Wissenschaftler seiner Generation bedienten: Selbst in Zeiten beruflicher Auslastung und wissenschaftlichen Engagements war der Physiker poetisch aktiv. Bemerkenswerter ist allerdings der Umstand, dass Schrödinger seine im weiteren Sinn >literarischen< Notizhefte, in denen er aus diversen Werken exzerpierte, Literaturlisten festhielt, kleine Bleistiftgemälde anfertigte, literarisch oder philosophisch dilettierte, keineswegs feinsäuberlich von seinen Arbeitsaufzeichnungen getrennt hielt. Während seine wissenschaftlichen Notizhefte und Mitschriften fallweise mit Bildern von nackten Frauen oder einzelnen Gedichten angereichert sind, bettet Schrödinger hier seine lyrischen Versuche in Kalkulationen und Skizzen zu einer »mathematischen Darstellungstheorie « ein. ${ }^{70}$ Der kreative Titel scheint sich also nicht nur auf die Hervorbringung von Literatur zu beziehen, sondern schließt auch die Genese von Wissen im Modus der Formel mit ein - mitunter auch in der heute nur noch schwer entzifferbaren GabelsbergerKurzschrift, die Schrödinger für die schnelle Entwicklung von Vortragsentwürfen oder das Festhalten von Ideen verwendete. Derartige tief bis ins Material reichende Amalgamierungen verschiedener Schreib- und Wissensformationen erinnern an epis-

69 Vgl. ÖZB Physik (o:143722), Sign. W33-785 (Heft 2) sowie W33-691.

70 Vgl. dazu allgemein z.B. Christoph Hoffmann, Schreiben im Forschen. Verfahren, Szenen, Effekte, Tübingen 2018. 
temische Notationspraktiken in der Tradition des Lichtenberg'schen $>$ Sudelns $<^{71}$ und führen über die auch bei anderen Physikern wie Werner Heisenberg oder Carl Friedrich von Weizsäcker nachweisbaren Reflexionen zum Verhältnis von Dichtung und Mathematik ${ }^{72}$ hinaus. Das betrifft auch Schrödingers gelegentliche »experiments in translation «, deren sich auch sein Dubliner Kollege, der Mathematiker John L. Synge, befleißigte und welche die naturwissenschaftliche Technik des Experiments für den linguistischen Zweck der Übersetzung fruchtbar machten. ${ }^{73}$ Auch wenn die konkrete Entstehungsgeschichte solcher Mappen aus den Nachlässen der betreffenden Physiker (noch) unklar ist, geben die überlieferten Zeugnisse Hinweise darauf, wie in der täglichen Arbeitspraxis literarische, physikalische und philologische Wissensformationen, Ästhetiken und Operationen nicht nur koexistieren, sondern mitunter symbiotisch verschmelzen konnten.

\section{V.}

\section{>Echte` Naturforscher? Das Bildungsmilieu der theoretischen Physik}

In seinem Aufsatz zur Milieubedingtheit der Naturwissenschaft sah Schrödinger den Zeitgeist als ein revolutionär-umstürzlerisches Bewusstsein in seiner eigenen Disziplin am Werk. Die Wissenschaftsgeschichte wiederum stellte seit den 1970erJahren esoterikaffine Gegenkulturen und wissenschaftsskeptische Strömungen der Weimarer Republik als besonders anschlussfähig für zentrale quantenphysikalische Denkmuster wie die Rede von der Aufhebung der Kausalität, der A-Logizität, der Irrationalität, Unschärfe und Unsicherheit alles möglichen Wissens über die Natur heraus. ${ }^{74}$ Wie die angeführten Beispiele deutlich gemacht haben dürften, wären solche Kulturalisierungen der modernen theoretischen Physik um eine Untersuchung der Interdependenzen von Physik und Philologie zu ergänzen. In diesem Zusammen-

\footnotetext{
71 Ausführliche Erläuterungen zu Traditionen >sudelnden< Notierens und ihrer epistemischen Relevanz finden sich in Petra McGillen, »Wit, bookishness, and the epistemic impact of note-taking. Lichtenberg's Sudelbücher as intellectual tools«, Deutsche Vierteljahrsschrift für Literaturwissenschaft und Geistesgeschichte 90 (2016), 501-528.

72 Vgl. z.B. einen Brief von Carl Friedrich von Weizsäcker an Erwin Schrödinger (30.05.1952), ÖZB Physik (o:143722), Sign. B33-947, oder Werner Heisenberg, »Ordnung der Wirklichkeit«, in: Gesammelte Werke. Abteilung C: Allgemeinverständliche Schriften. Band I: Physik und Erkenntnis 1927-1955, hrsg. Walter Blum, Hans-Peter Dürr, Helmut Rechenberg, München 1984, 217-306, hier: 222-226.

73 Synges Aufsatz »An Experiment in Translation« (1957) mit Schrödingers handschriftlichen Annotationen wird an der ÖZB für Physik verwahrt (Nachlass Schrödinger, Kasten 21). Schrödingers Sprachbewusstsein zeigt sich nicht zuletzt daran, dass er etwa bei der Drucklegung seines Beitrages zu »Louis de Broglie, Physicien et Penseur« darauf Wert legte, dass »neben der französischen Übersetzung auch seine englische Originalfassung abgedruckt wurde, und zwar auf gegenüberliegenden Seiten!« Vgl. Gabriele Kerber, Wolfgang Kerber: Nachwort und Hinweise für den Benutzer, in: Erwin Schrödinger, Gesammelte Abhandlungen. Band 4: Allgemein wissenschaftliche und populäre Aufsätze, hrsg. Österreichische Akademie der Wissenschaften, Wien 1984, 615-616, hier: 616.

74 Es soll nicht unerwähnt bleiben, dass derartige quantenphysikalische Paradigmen in gewisser Spannung mit der Wissenseuphorie und Erkenntnisgewissheit standen, die mit der Entwicklung der Quantentheorie einhergingen und die bspw. auch in Schrödingers populären Publikationen oder dem Schrödinger'schen Schreiben an Cassirer fassbar sind.
} 
hang interessiert freilich weniger der soziokulturelle Rahmen jener Zeit, in der die Quantentheorie formuliert wurde, als vielmehr das konkrete Bildungsmilieu, in dem die Quantenphysiker selbst sozialisiert wurden: Die überwiegend deutschsprachigen Begründer der Quantentheorie stammten fast ausnahmslos aus dem Bildungsbürgertum. Der Besuch eines prestigeträchtigen humanistischen Gymnasiums war für sie eine Selbstverständlichkeit. ${ }^{75}$

In den renommierten Bildungsinstitutionen kam der philologischen Schulung ein bedeutend höherer Stellenwert zu als der mathematisch-naturwissenschaftlichen ${ }^{76}$ und dort konnten Heisenberg, Schrödinger, Pauli, Born und ihre Kollegen, so sie noch nicht familiär bedingt oder gar in professoralen Haushalten philologisches Wissen quasi mit der Muttermilch aufgesogen hatten, ${ }^{77}$ schon früh in Kontakt mit den Alten Sprachen und grundlegenden philologischen Techniken kommen. Die durchgängige Hochschätzung dieser $>$ philologischen Schule $<,{ }^{78}$ welche die deutschsprachigen theoretischen Atomphysiker durchlaufen hatten, ist in Egodokumenten wie späteren Reden anlässlich von Jubiläumsfeiern der besuchten Gymnasien oder Korrespondenzen auf vielfältige Weise dokumentiert. In einem an den Direktor des Wiener Akademischen Gymnasiums gerichteten Brief, abgefasst anlässlich der 400Jahr-Feier der Schule, bringt Schrödinger seine Dankbarkeit gegenüber der Bildungsanstalt zum Ausdruck und hebt insbesondere die Relevanz des altphilologischen Unterrichts hervor: »wie gerne möchte ich all Ihren Schülern einen vollen Eindruck davon geben, was die Einführung in die Antike und die Erlernung der alten Sprachen im späteren Leben für mich bedeutet hat und noch bedeutet, obwohl sie für mein engeres Fachgebiet - die theoretische Physik - gar nicht >notwendig< zu sein scheinen. Es ist eine Erweiterung des Lebensraums, ohne die ich mich arm fühlen würde. ${ }^{79}$ Derartige Elogen finden sich bei vielen Atomphysikern, nicht selten in expliziter Beziehung auf ihre spätere Tätigkeit als Naturwissenschaftler. So hält Max Laue als Rezept zur Förderung des wissenschaftlichen Nachwuchses, insbesondere des naturwissenschaftlichen, fest: »Schickt die Jungens ins Gymnasium

\footnotetext{
75 Hier wäre auch auf ein nationales Spezifikum zu verweisen, stammte doch ein großer Teil der führenden Vertreter der frühen Quantentheorie aus dem deutschsprachigen Raum, wo der Ausbildung an humanistischen Eliteanstalten ein höheres Renommee zukam als etwa im angelsächsischen Raum. Es dürfte kein Zufall sein, dass Physiker mit einem anderen Bildungshintergrund wie etwa Paul Dirac für ihre Formelaffinität bekannt sind, das Problem einer Sprache der Quantentheorie hingegen von den deutschen Kollegen besonders engagiert diskutiert wurde.

76 So kam z.B. Werner Heisenberg am elitären Münchener Maximiliansgymnasium am Beginn seiner schulischen Laufbahn in den Genuss von acht Stunden Latein und sechs Stunden Griechisch wöchentlich, wohingegen nur vier Stunden Mathematik unterrichtet wurden. Ähnliche Stundenverteilungen sind auch für das Wiener Akademische Gymnasium überliefert, an dem Erwin Schrödinger unterrichtet wurde. Vgl. David C. Cassidy, Werner Heisenberg. Leben und Werk, Heidelberg, Berlin 2001, 43; Moore (Anm. 60), hier: 29.

77 Werner Heisenbergs Großvater Nikolaus Wecklein beispielsweise war promovierter Altphilologe und Rektor des Münchener Maximilians-Gymnasiums; sein Vater August Heisenberg war Byzantinist. Vgl. Cassidy (Anm. 76), hier: v.a. 22-28.

78 Sheldon Pollock, Philologie und Freiheit, Berlin 2016, hier: 43.

79 Zit. nach Kerber, Dick, Kerber (Anm. 63), hier: 20. Und weiter: »Es gibt einem das Gefühl der Einheit mit den großen Denkern des Altertums, von Thales und Demokrit bis zu Archimedes und Ptolemäus, ... Und wenn ich im Boethius lese, ... dann weiß ich, was ich am Akademischen Gymnasium gehabt habe!«.
} 
und lasst sie dort die alten Sprachen gründlich treiben! « ${ }^{80}$ Während so mancher nach 1945 geborene Physiker infolge der sukzessiven Ablösung der humanistischen Gymnasien durch andere, zielgerichtetere Wege zur Universitätsreife gar nicht mehr in Berührung mit den Alten Sprachen gekommen sein mag, könnte den Gründervätern der Quantenphysik die Ausprägung einer charakteristischen >scientific persona ${ }^{81}$ attestiert werden, welche die humanistisch-philologische Gelehrsamkeit als zentralen Bestandteil der eigenen wissenschaftlichen Praxis begriff.

Die schon von Kindesbeinen an bestehende Verbindung zu den Geisteswissenschaften, insbesondere den Sprach- und Textwissenschaften, konnten die Physiker auch zu Zeiten aufrecht halten, in denen sie schon längst über ihre gymnasiale Ausbildung hinausgewachsen waren und ihre Berufung gefunden hatten: in professoralen Netzwerken, die einen gleichermaßen produktiven wie interdisziplinären Wissensaustausch ermöglichten. Zu diesen Netzwerken zählten zunächst größere institutionalisierte Zusammenhänge wie die Akademien. So ist in den Sitzungsberichten der Preußischen Akademie der Wissenschaften, in der auch Schrödinger Mitglied war, bis er nach der Machtergreifung Hitlers seine Mitgliedschaft freiwillig niederlegte, dokumentiert, dass z.B. Einstein oft und gerne den Vorträgen der Philologen, etwa Wilamowitz-Moellendorffs oder Hermann Diels', lauschte: Der Physiker erläuterte in just jener Sitzung die Perihel-Bewegung des Merkur mittels seiner Allgemeinen Relativitätstheorie, in der Diels über seine Nachkonstruktion von Platons Nachtuhr berichtete (welche er übrigens mithilfe seines Sohnes, des Chemikers und späteren Nobelpreisträgers Otto Diels, angefertigt hatte). ${ }^{82}$ Solch frappante Konstellationen dürften maßgeblich dazu beigetragen haben, dass eine von Diels angefertigte Übersetzung des Lukrez'schen Lehrgedichts De Rerum Natura mit einer Einleitung von keinem Geringeren als Einstein erscheinen und vom Physiker und Chemie-Nobelpreisträger Walther Nernst rezensiert werden konnte. ${ }^{83}$ Erwin Schrödinger selbst stellte in seinem (übrigens ebenfalls in einer Akademie-Sitzung gehaltenen) Vortrag »Ist die Naturwissenschaft milieubedingt? « interdisziplinäre Kontaktzonen als wichtige Schaltstellen im wissenschaftlichen Arbeitsprozess heraus: Nicht nur konnte der Forscher hier innerhalb eines zwar universitär geprägten, so doch fachübergreifenden sozialen Umfelds mit aktuellen Strömungen seiner Zeit in Kontakt geraten und sich bei seiner Arbeit davon inspirieren lassen. Daneben fungierten die Akademien als Kontrollinstanzen, welche die eigenen Forschungen mitunter neu perspektivierten, den Wissenschaftler dadurch anregten, sich der Relevanz seiner Arbeit zu vergewissern bzw. seine Fragestellungen gegebenenfalls anzupassen:

\footnotetext{
${ }^{80}$ Max von Laue, »Mein physikalischer Werdegang. Eine Selbstdarstellung«, Aufsätze und Vorträge, Braunschweig 1962, VII-XXXVI, hier: IX. Vgl. auch Heisenbergs Lob des humanistischen Gymnasiums in M. Haußner, »Interview mit Prof. Heisenberg«, Maximiliansgymnasium München. Bericht über das Schuljahr 1972/73 und Stimmen aus dem Maxgymnasium (1973), 14-17.

81 Lorraine Daston, H. Otto Sibum, »Introduction. Scientific Personae and Their Histories«, Science in Context 16/1-2 (2003), 1-8.

82 Sitzungsberichte der Königlich Preußischen Akademie der Wissenschaften (18.11. 1915), 803.

83 Vgl. dazu Wolfgang Rösler, »Hermann Diels und Albert Einstein. Die Lukrez-Ausgabe von 1923/24«, in: William M. Calder, III, Jaap Mansfeld (Hrsg.), Hermann Diels (1848-1922) et la science de l'antiquité, Genf 1999, 261-294.
} 
Beiläufig bemerkt, es scheint mir eine der fruchtbarsten und heilsamsten Funktionen solcher Körperschaften, in denen Forscher aus allen Gebieten zu gemeinsamer Arbeit sich vereinigen, daß der Einzelne sich gelegentlich besinnen muß, daß er über seine eigentlichen Ziele und Triebfedern Auskunft geben muß vor Männern, die er respektiert und ganz voll nimmt und bei denen er nicht in Gefahr kommt, sie mit einer schnellen Verlegenheitsantwort abzuspeisen, in dem hochmütigen Gedanken: der kann das ja doch nicht verstehen. ${ }^{84}$

Wie auch der Beginn von Schrödingers Brief an Cassirer deutlich macht, demzufolge der Physiker seinen Kollegen nicht einfach mit einem »unsachlichen, förmlichen, rein conventionellen Dank« abspeisen, sondern die »Dinge erst richtig durchdenken« wollte, um »etwas irgendwie wesentliches darüber schreiben zu können«, nahm Schrödinger den interdisziplinären Austausch durchaus ernst, ja betrachtet ihn als wichtiges Instrument im Prozess der Wissenserzeugung. ${ }^{85}$

Möglichkeiten zu solchem Austausch boten sich nicht nur in den großen Akademien, sondern auch im privaten Rahmen - etwa in Professorenzirkeln wie der berühmten Berliner Mittwochsgesellschaft oder kleineren Vereinigungen wie dem Leipziger Professorenkränzchen »Coronella «. ${ }^{86}$ Lockere Freizeitaktivitäten und abendliche Zusammenkünfte im häuslichen Umfeld brachten die Mitglieder miteinander in Kontakt und schufen Raum für universitätspolitische Gespräche, Probevorträge und interdisziplinäre Diskussionen. Als »Coronella «-Mitglied konnte der junge Professor Werner Heisenberg beispielsweise akademische Bekannt- und Freundschaften mit Figuren wie dem Altertumswissenschaftler Helmut Berve, den Philologen Friedrich Klingner und Wolfgang Schadewaldt oder Hans Georg Gadamer pflegen, dessen Berufung nach Leipzig nicht zuletzt auf Heisenbergs Interesse an Gadamers Untersuchungen zur antiken Atomistik zurückzuführen war. ${ }^{87}$ Daneben bot zur damaligen Zeit auch das allgemeine gesellige Leben der Professorenfamilien Raum für fachliche, philosophische oder musikalische Begegnungen. So verkehrte Schrödinger als Arbeitskollege des jungen Physikers Max Delbrück, Sohn des Historikers Hans Delbrück, während seiner Berliner Zeit gerne in dessen Familienhaus, wo sich immer wieder Möglichkeiten fachübergreifender Kontakte ergeben konnten. Beim Ehepaar Planck wiederum lauschte man in gelehrter Atmosphäre Kammermusikkonzerten. ${ }^{88}$ Und im Hause Schrödinger fanden die beliebten »Würstelabende « statt, zu denen neben einem breiten naturwissenschaftlichen Kollegium auch diverse private Bekanntschaften erschienen - im Gästebuch unterzeichnete als

\footnotetext{
84 Schrödinger (Anm. 27), hier: 304.

85 Vgl. dazu auch die euphorische Rezension Schrödingers zu Arthur S. Eddingtons Ende der 1920erJahre erschienenem Buch The Nature of the Physical World, in der Schrödinger metaphernreich die Rolle der Wissenschaftspopularisierung im Forschungsprozess herausstreicht. Erwin Schrödinger, »Eddington, A. S., The Nature of the Physical World. Gifford Lectures 1927«, Die Naturwissenschaften 17 (1929), 694.

86 Gerald Wiemers, »Werner Heisenberg und die Leipziger Professoren-Vereinigung >Coronella «, in: Christian Kleint, Helmut Rechenberg, Gerald Wiemers (Hrsg.), Werner Heisenberg 1901-1976. Beiträge, Berichte, Briefe. Festschrift zu seinem 100. Geburtstag, Stuttgart, Leipzig 2005, 141-145.

87 Hans-Georg Gadamer, »>Die Griechen, unsere Lehrer $<$. Ein Gespräch mit Glenn W. Most«, Internationale Zeitschrift für Philosophie (1994), 139-149.

88 Kerber, Dick, Kerber (Anm. 63), hier: 77.
} 
»stille Zuschauerin « etwa Mira Koffka, ${ }^{89}$ bei der es sich um eine Stieftochter Georg Bondis und spätere Verlagsmitarbeiterin sowie Übersetzerin von Schrödingers englischsprachigen Vorträgen (Nature and the Greeks) handeln dürfte. Zu Gast gebeten wurde man übrigens einigermaßen poetisch, wie das gereimte Einladungsschreiben zeigt $^{90}$ - auf das Physiker/innen wie Max Planck oder Lise Meitner in ähnlich ambitionierten Versen antworteten. ${ }^{91}$

Ruft man sich nun noch einmal Erwin Schrödingers brieflich geäußerte Behauptung in Erinnerung, dass der »echte[] Naturforscher von heute [...] eine tiefe Verachtung gegen Literatur « hege, so regen sich nicht nur starke Zweifel, ob und inwiefern der Verfasser sich selbst überhaupt dem Kreis moderner Forscher zugeschlagen hat. Angesichts der offensichtlichen literarisch-philologischen Inklinationen der theoretischen Physiker könnte man auch fragen: Wären all diese Wissenschaftler in ihrer Liebe zur Literatur dann unzeitgemäß gewesen? Oder fügten sie sich mit ihrer Neigung in einen zeitgenössischen Trend - wären im Sinne Schrödingers aber womöglich gar nicht als >echte Naturforscher $<$ zu bezeichnen?

\section{VI.}

\section{Feine Unterschiede}

Die bisherigen Ausführungen dürften deutlich gemacht haben, dass für eine adäquate Einordnung von Schrödingers Brief an Cassirer sowie der darin vermeintlich zum Ausdruck kommenden abschätzigen Haltung gegenüber >Literatur $<$ Differenzierungen notwendig sind - in mehreren Richtungen. So ist zunächst von Pauschalisierungen wissenschaftlicher Disziplinen Abstand zu nehmen. Wenn Schrödinger in seinem Schreiben die Haltung >der Naturforscher im Allgemeinen referiert und die Frage nach der Milieubedingtheit >der < Naturwissenschaft mit einer kulturellen Verortung >der< Physik beantwortet, insinuiert er die Homogenität ganzer Disziplinen bzw. fakultärer Einheiten. Das mag in einem polemischen Privatbrief oder einer essayistischen Skizze ohne systematischen Anspruch durchaus sinnvoll sein. Aus analytischer Perspektive ist freilich zu beachten, dass mit solcherlei Generalisierungen Verbindungslinien verwischt werden, die sich auf subdisziplinärem Level präzise herausarbeiten ließen. Eine aussagekräftige Untersuchung von Wechselbeziehungen

\footnotetext{
89 Plakat der ÖZB für Physik, Wien, Original im Besitz der Familie Braunizer.

90 »Ihr wißt, wir haben euch versprochen / Am 4. wieder Würstel zu kochen! / Wir lieben die Abwechslung, drum zum Beweis / Sind diesmal die Würstel nicht braun sondern weiß. / Dazu gibt's wie immer Bretzeln und Bier / Und bleiben könnt Ihr bis gegen vier. / Neu ist der Name der Lokalität / und das ist Ehrenfests Priorität: / Ihr seid geladen, verehrte Damen und Herrn / in die Cunostraße ins Hotel $\psi \psi^{*}$. «Zit. nach Kerber, Dick, Kerber (Anm. 63), hier: 78.

91 So reimte das Ehepaar Planck: »Wenn Ihr Euch wirklich nicht scheut, / Einzuladen solch alte Leut, / So wird auch das Ehepaar Planck / Bei Euch erscheinen mit vielem Dank. / Denn wir kommen ja so gern / In das moderne Hotel $\psi \psi^{*} \ll$. Lise Meitner dichtete: »Bei Schrödingers ist Würstelabend, wer ging nicht gerne hin, / Wo sicher frohe Stimmung herrscht und wo man trifft halb Wien / Der böse Zufall aber will's, daß ich nicht kommen kann, / weil ich an diesem Abend muß, den Vortrag hören an, / den Born hält vor der Chemie (und ich im Vorstand bin). / Bei Schrödingers ist Würstelabend und ich, ich kann nicht hin!« Zit. nach Kerber, Dick, Kerber (Anm. 63), hier: 78.
} 
zwischen Physik und Literatur um 1900, die über die Konstatierung vager Analogien hinausgeht, erfordert also die Berücksichtigung kleinteiliger Entwicklungsund Ausdifferenzierungsprozesse, etwa mit Blick auf die Ablösung der Vormachtstellung der Experimentalphysik durch die Theorie. Außerdem ist, gerade im Fall der modernen Atomphysik mit ihren aufgeheizten Debatten, auch der disziplininternen Vielfalt an Positionen und Weltbildern Rechnung zu tragen - die ihrerseits wiederum, selbst bei ein und derselben Forscherpersönlichkeit, in Abhängigkeit von publizistischem Kontext, Adressaten oder auch der momentanen individuellen Lebenssituation durchaus variieren konnten. Von >der< Antikefaszination Schrödingers wäre insofern also genauso wenig zu sprechen wie von einer Literaturbegeisterung (oder -verachtung) >der $<$ Physik. Der Brief an Cassirer für sich erlaubt dementsprechend auch keine generalisierbare Aussage über das komplexe Beziehungsgefüge von Literatur und Physik: Mit Blick auf den polemischen Tonfall sind neben dem bereits diskutierten Überdruss an zeitgeist-typischen soziokulturellen bzw. philosophischen Deutungen der modernen Physik eben auch persönliche Befindlichkeiten Schrödingers und inhaltliche Differenzen zu den in Cassirers Indeterminismus-Studie vertretenen Positionen in Rechnung zu stellen, die im Lauf der Zeit starken Schwankungen unterworfen sein konnten. ${ }^{92}$ Beispielhaft hierfür wäre die recht ausführliche briefliche Kritik an Cassirers Rekurs auf Schrödingers Wiener Lehrer Franz Exner: Dieser hatte mit Blick auf die statistische Thermodynamik bereits Jahre vor der Entwicklung der Quantenmechanik das Kausalgesetz spekulativ infrage gestellt. Schrödinger sah seinen Lehrer und dessen Intuitionen gegenüber Heisenberg, der derartige philosophische Konzeptionen mit seiner Mechanik und seinen Unschärferelationen auf ein formelhaftes Fundament gestellt hatte, bei Cassirer offenbar zu wenig gewürdigt - womöglich auch seine eigenen, auf Exner aufbauenden physikalisch-philosophischen Spekulationen, die er in seiner Züricher Antrittsvorlesung 1922 geäußert hatte, später freilich, nach seiner >(Rück-)Wende < zu einer Physik der anschaulichen Modelle, geflissentlich ignorierte.

$\mathrm{Zu}$ beachten ist, dass sich umgekehrt aus solchen Dissonanzen keineswegs auf eine ablehnende Grundhaltung Schrödingers gegenüber Cassirer schließen lässt zu berücksichtigen wäre hier u.a. auch die Tradition des polemischen Gelehrtenaustauschs, an die Schrödinger anknüpft. Jahre später jedenfalls ist der längst verstorbene Cassirer dem Physiker in seinem Lebensabriss, ${ }^{93}$ aber auch in der Kommunikation mit Fachkollegen als Gewährsmann willkommen - beispielsweise in einem Brief an Max Born, in dem Schrödinger wie so oft gegen das Komplementaritätsprinzip wettert. Das von Bohr aufgestellte und von Schrödinger stets kritisch reflektierte Postulat, demzufolge (u.a.) eine Darstellung in der Raum-Zeit bei gleichzeitiger Erfüllung des Kausalitätsprinzips einander ausschlössen (Raum-Zeit-Darstellung und Kausalitätsforderung einander also »komplementär« wären), wurde von Bohr selbst schon früh verallgemeinert und im Sinne einer »naturwissenschaftlich « fundierten Begründung der Willensfreiheit eingesetzt - sehr zum Missfallen Schrödingers:

\footnotetext{
92 Vgl. dazu auch Ryckman (Anm. 7).

93 Erwin Schrödinger, Mein Leben meine Weltansicht, Zürich 1989, hier: 44.
} 
Und nun gar: das ur-ur-alte Rätsel freier Wille gegen kausale Determiniertheit habe nun endlich seine Lösung gefunden durch die gloriose Erfindung der Komplementarität. (Schüler: Doch ein Begriff muß bei dem Worte sein. Mephisto: Schon gut, nur muß man sich nicht allzu ängstlich quälen, Denn eben wo Begriffe fehlen, da stellt ein Wort zur rechten Zeit sich ein. Ich könnte die Stelle vollständiger hersetzen, obwohl ich das Buch nicht mithabe aber ich will mich jetzt im schönen Zug des Schimpfens nicht länger unterbrechen.)

Ja habt Ihr denn wirklich keine Ahnung wie naiv Ihr seid? Liest niemand den Ernst Cassirer oder wenigstens mich? Kennt niemand das erste Buch des Lucretius Carus? welches - oder vielleicht sein Held Epikur das Rätsel von [sic! es dürfte sich hierbei um einen Transkriptionsfehler handeln: vor] 2000 oder mehr Jahren schon so ziemlich auf dieselbe Art gelöst hat, bloß mit weniger Stacheldraht von Formeln, welcher es heute den armen unschuldigen Lämmern von Offizieren der Bundeswehr aufzumutzen erlaubt. So Dixi. ${ }^{94}$

Gespickt mit Referenzen auf Texte wie das Lukrez'sche Lehrgedicht - in den 1920er-Jahren von Hermann Diels neu übersetzt ${ }^{95}$ - ist die Passage selbst ebenso vielschichtig wie der nur vermeintlich simple Brief an Cassirer. Doch während Schrödinger in seiner Korrespondenz mit dem Philosophen eine gewisse Zurückhaltung gegenüber geisteswissenschaftlichen Wissenstechniken zur Schau stellt und insbesondere die Aktualitätseuphorie der Naturwissenschaftler und ihre Fähigkeit zur Selbstbeglaubigung durch die zentrale Technik der Formel zumindest auf den ersten Blick eher positiv zu bewerten scheint, nimmt der »schöne[] Zug des Schimpfens« hier eine etwas andere Wendung. Bohrs formelbewehrte Anhänger situiert Schrödinger im Brief an den Freund geradewegs in der Tradition einer philosophischen »Hexenküche $«,{ }^{96}$ in der schon, wie er heraushebt, vor über 2000 Jahren ein ganz ähnliches Süppchen gekocht wurde. Wenn Schrödinger an anderer Stelle die »Schärfe « beeindruckt, »mit der sich schon dem frühesten [i.e. vorsokratischen!, MMG] naturwissenschaftlichen Denken die Unerbittlichkeit der Kausalforderung abhob $\ll,{ }^{97}$ so sieht er die Reflexionen seiner Zeitgenossen zum Problem der Willensfreiheit als abgestandenen Aufsud der von Lukrez überlieferten Epikureischen Ideen: »behufs Rettung der Freiheit des Willens« habe der griechische Philosoph vorgeschlagen, »die absolute Gesetzlichkeit der Atombewegungen kleinweise aber ohne Unterlaß durchbrochen $[\mathrm{zu}]$ denken $\ll{ }^{98}$ Die Auflösung des Widerspruchs zwischen Determinismus und Willensfreiheit auf Basis des Komplementaritätsprinzips - durch das etwas penetrante Faust-Zitat als nachgerade teuflische Sophisterei diffamiert - ist für Schrödinger also nicht viel mehr als ein Abklatsch der von ihm hier sehr kritisch bewerteten Gedanken des Epikur, die durch einen »Stacheldraht von Formeln« vor den Einwänden Fachunkundiger abgeschirmt werden.

\footnotetext{
94 Brief von Erwin Schrödinger an Max Born (10.10.1960), zit. nach Meyenn (Anm. 4), hier: 734.

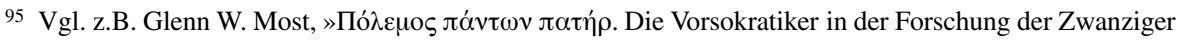
Jahre«, in: Hellmut Flashar (Hrsg.), Altertumswissenschaft in den 20er Jahren, Stuttgart 1995, 87-114.

96 Schrödinger an Cassirer (Anm. 4).

97 Schrödinger (Anm. 44), hier: 48.

98 Schrödinger (Anm. 44), hier: 47.
} 
Auffällig ist an solchen Passagen nicht zuletzt der naturwissenschafts- und erkenntnisskeptische Grundtenor Schrödingers, welcher dem zentralen Symbolsystem seiner Disziplin, der Formel, zumindest misstrauisch, wenn nicht kritisch gegenübersteht. Schon Friedrich Nietzsche hat im ausgehenden 19. Jahrhundert, der Hochphase des >Age of Scienceく, wiederholt alles andere als wertfreie Überlegungen zur Physik und ihren Prinzipien angestellt: Wiewohl Nietzsche der Physik zumindest fallweise, etwa in der Fröhlichen Wissenschaft, durchaus wohlgesonnen zu sein scheint, mitunter gar ein »Hoch« auf das im Aufschwung begriffene Fach und seine Methode der genauen Beobachtung ausbrachte, ${ }^{99}$ qualifizierte er es an anderen Stellen, insbesondere in den nachgelassenen Fragmenten, massiv ab. Als Disziplin emsiger Maschinisten und Ingenieure, welche sich »auf den Glauben an die Sinne stellt«, »Augenschein und Handgreiflichkeit für sich« hat und deshalb »bezaubernd, überredend, überzeugend « auf ein »Zeitalter mit plebejischem Grundgeschmack « wirke, ${ }^{100}$ als Leitwissenschaft seiner Zeit, die ihre tatsächliche »Welt-Auslegung « zur »WeltErklärung « hypostasiere, verortete er die Physik in einem spezifischen Setting von Technik-, Kulturkritik und Massenkritik, welches das 20. Jahrhundert variantenreich durchdeklinieren würde. ${ }^{101}$ Vor diesem Hintergrund gewinnt denn auch der auf den ersten Blick geisteswissenschaftlich-literarischen Wissensformationen gegenüber abfällige Brief Schrödingers an Cassirer eine etwas andere Note. An genau solche naturwissenschaftskritischen Denkmuster knüpft nämlich auch der Physiker an, wenn er an Cassirer schreibt: »Wir Naturwissenschaftler sind Parvenus, Nouveau-riches. Dies ist unaufrichtig gesprochen, denn selbst bezeichnet man sich nicht gern mit so abfälligen Namen. Nouveau-riche ist dritte Person. Die erste Person lautet: Self-made-man. Wir sind eine Art Bolschewiken in der Wissenschaft und lieben nicht die Beziehung auf jene Gesellschaftsordnung, die der unseren vorherging. «102

In Anbetracht solch despektierlicher Titulierungen verwundert es wenig, dass Schrödinger explizit seine eigene Gruppenzugehörigkeit thematisiert. Realiter dürfte sich der ein elitäres kontinentales Selbstverständnis pflegende Altösterreicher mit Bolschewiken oder kapitalistischen Neureichen, die sich als ungebildete Empor-

\footnotetext{
99 Friedrich Nietzsche, Die fröhliche Wissenschaft (1882), § 335, zit. nach eKGWB/FW-335. Bezeichnend für Nietzsches Hochschätzung der Physik ist auch die Tatsache, dass er Ernst Mach persönlich zwar nicht kannte, ihm indes (wie einem sehr selektiven Kreis an weiteren Naturforschern) ein Exemplar seiner teuer verlegten »Genealogie der Moral« übersandte (wie umgekehrt die ausbleibende bzw. eher förmliche Reaktion der Naturwissenschaftler bezeichnend ist - Mach war der einzige (!), von dem eine solche überliefert ist, und zwar in Form der Zusendung eines Aufsatzes über »die photographische Fixierung der durch Projektile in der Luft eingeleiteten Vorgänge «). Vgl. dazu auch Helmut Heit, »Nietzsches Philosophie und das >Age of Science««, in: Helmut Heit, Lisa Heller (Hrsg.), Handbuch Nietzsche und die Wissenschaften. Natur-, geistes- und sozialwissenschaftliche Kontexte, Berlin, Boston 2014, 19-45, hier: 40-41.

100 Friedrich Nietzsche, Jenseits von Gut und Böse (1886), § 14, zit. nach eKGWB/JGB-14.

101 Vgl. dazu allgemein etwa Georg Bollenbeck, Eine Geschichte der Kulturkritik. Von Rousseau bis Günther Anders, München 2007, sowie Kapitel 3 und 4 in Magdalena Gronau, Kritische Essayistik zwischen den Two Cultures. Das »zweite Leben« des Biochemikers Erwin Chargaff, Innsbruck 2019. Beispielhaft sei auf die Technik- und Naturwissenschaftskritik von Friedrich Georg Jünger, Günther Anders oder Erwin Chargaff verwiesen: Günther Anders, Die Antiquiertheit des Menschen. Über die Seele im Zeitalter der zweiten industriellen Revolution, München 1956; Erwin Chargaff, Das Feuer des Heraklit. Skizzen aus einem Leben vor der Natur, Stuttgart 2002; Friedrich Georg Jünger, Die Perfektion der Technik, Frankfurt a.M. 2010.

102 Schrödinger an Cassirer (Anm. 4).
} 
kömmlinge jeden Trend eilig zu Nutze machen und von einer umfassenden Verachtung gegenüber allem Unzeitgemäß-Vergangenem beseelt sind (dabei jedoch hochmütig ihre eigene Traditionsgebundenheit übersehen), ebenso wenig identifiziert haben wie mit den formelversessenen »Offizieren der Bundeswehr «, die er in seinem wütenden Brief an Max Born abkanzelt. ${ }^{103}$ Dass Schrödinger sich gerade im Briefwechsel mit Fachkollegen nicht selten als gebildeter Gegenpol einer durch zu viel stupide Mathematik banalisierten, sich in leeren Wort- und Formelhülsen verlierenden neuen Physik in Szene setzt und seine Außenseiterposition in der Riege der Gründerväter der Quantentheorie heraushebt, lässt den interdisziplinären Austausch mit Cassirer in anderem Licht erscheinen: Schrödingers ruppiger Ton dürfte nicht zuletzt Ausdruck seiner Enttäuschung sein - der Enttäuschung darüber, dass eben selbst ein Philosoph wie Cassirer »dem unendlich angeschwollenen Tam-Tam der modernen Quantenphysik « aufsitzt und viel zu unkritisch die Banalitäten einer, zumindest aus Schrödingers Sicht, ebenso durchrationalisierten wie laienphilosophisch aufgeplusterten Disziplin für epistemologische Großtaten hält. Insofern ist Schrödingers Brief eben nicht nur lesbar als Kritik an geisteswissenschaftlich-literarischen Wissensformationen, sondern auch und vor allem als Warnung - vor falscher Hochachtung vor der formelbewehrten >Philosophie der (Quanten)Physiker $<.{ }^{104}$

\section{VII.}

\section{Nachtrag}

Wenn Nietzsche im späten 19. Jahrhundert über die Physik nachdachte, so hatte er dabei die seinerzeit dominante Experimentalphysik im Blick. Von den tiefgreifenden Veränderungen, welche die Epochenschwelle um 1900 mit sich brachte, konnte er nichts wissen: Der bis dato wenig geliebten theoretischen Physik gelang es, sich innerhalb weniger Jahre in Deutschland als Speerspitze eines Faches zu etablieren, dem gerade die von Nietzsche elitistisch verachtete Sinnlichkeit und »Handgreiflichkeit«, präziser: seine Anschaulichkeit zu großen Teilen abhandenkam. ${ }^{105}$ Anknüpfend daran lassen sich die aufgezeigten textuellen und institutionellen Verschränkungen von

\footnotetext{
103 Hier spielt er aller Wahrscheinlichkeit nach auf eine sich den Nationalsozialisten anbiedernde Physik an, für die stellvertretend der mathematisch besonders begabte Pascual Jordan stehen könnte. Jordan hatte gemeinsam mit Werner Heisenberg und Max Born in Göttingen die sogenannte »Drei-Männer-Arbeit « verfasst, welche nur kurz vor Schrödingers Publikation der Wellenmechanik mit der hoch komplexen, unanschaulichen Matrizenmechanik einen mathematischen Formalismus zur Lösung quantenphysikalischer Probleme lieferte.

104 Was er von den Dilettantismen seiner Fachkollegen hält, macht auch der letzte Satz aus Schrödingers letztem Brief an den Freund Max Born mehr als deutlich. Am 24.10.1960 warnt er den Freund in deutlichen Worten vor philosophischen Spekulationen über Quantentheorie und Willensfreiheit: »Damit gibst Du uns dem Gespött der Philosophen preis, so daß sie, wenn ein Physiker (über) Philosophie redet, nicht mehr hinhören. « Bezeichnend ist insbesondere die Fußnote, die diesem Satz folgt: »Ich ja auch nicht. Aber die alten Vorträge von Max Planck waren fein.«Zit. nach Meyenn (Anm. 4), hier: 741.

105 Einführende Erläuterungen zur Unanschaulichkeit der Quantenphysik sowie detaillierte Ausführungen zu Erwin Schrödingers Verständnis einer anschaulichen Physik in Gronau, Gronau (Anm. 17).
} 
Physik und Philologie epistemologisch und selbst praxeologisch weiterspinnen: ${ }^{106}$ Die Aufgabe der Theorie verschob sich dahin, experimentell festgestellte Abweichungen von gängigen Modellen in einem konsistenten mathematischen Formalismus zu harmonisieren, diesen zu verfeinern und schließlich - ausgehend von der zugrunde liegenden Mathematik - praktische Ergebnisse zu prognostizieren, welche erst im Anschluss in eigens für den Nachweis designten Experimentalsettings bestätigt wurden. ${ }^{107}$ Die Theorie begann, dem Experiment vorauszulaufen. Der theoretische Physiker jener Zeit bezog sich in diesem Zusammenhang nicht mehr nur auf unmittelbar mit den Sinnen wahrnehmbare Erscheinungen, sondern auf deren zu Papier gebrachte Spuren, ${ }^{108}$ im weiteren Sinn: auf Texte, wie er sie ja auch selbst produzierte. Nicht unähnlich dem klassischen Editionsphilologen zielte er darauf ab, bruchstückhafte, fallweise widersprüchliche >Quellen< genau zu lesen, zu bewerten, zu einem sinnhaften Ganzen zu verbinden und mithilfe des von ihm generierten Regelwerks noch nicht aufgefundene Belege vorherzusagen, deren Existenz erst später nachzuweisen sein würde.

Geblieben war freilich auch der Subdisziplin der theoretischen Physik, wiewohl sie gerade in Hinblick auf ihre Praktiken, Operationen, Orte und Dinge der Wissensproduktion mit der Experimentalphysik nicht in einen Topf zu werfen ist, die von Nietzsche so geschätzte zentrale Technik der genauen Beobachtung, die dieser nicht nur an der Physik bewunderte, sondern insbesondere an seiner eigenen Herkunftsdisziplin. Ungeachtet Nietzsches Geringschätzung des zeitgenössischen Philologenstandes erscheint bei ihm nämlich just die Philologie als unzeitgemäße Wissenschaft der langsamen, tiefen Lektüre und Beobachtung, der differenzierten Abwägung und grundlegenden Zurückhaltung im Urteilen, für die er den Begriff der »Ephexis « prägte. ${ }^{109}$ Diese basalen philologischen Techniken des aufmerksamen Lesens und abwägenden Urteilens waren gerade den Schöpfern der modernen theoretischen Physik im Zuge ihrer profunden altphilologischen Ausbildung in Fleisch und Blut übergegangen. Nimmt man Sheldon Pollocks Diktum, demzufolge »[f]ür jemanden, der durch diese Schule gegangen ist, [...] Philologie zum Rüstzeug für

106 Anregend hierzu ist der programmatische Aufsatz von Lorraine Daston, Glenn W. Most, »History of Science and History of Philologies «, Isis 106/2 (2015), 378-390. Zu einer Praxeologie der Geisteswissenschaften, die sich in weiteren Teilen auf die Schreibtischwissenschaft der theoretischen Physik übertragen lassen, vgl. auch Carlos Spoerhase, »Das >Laboratorium < der Philologie?«, in: Andrea Albrecht, Lutz Danneberg, Olav Krämer, Carlos Spoerhase (Hrsg.), Theorien, Methoden und Praktiken des Interpretierens, Berlin, München, Boston 2015, 53-80; Carlos Spoerhase, Mark-Georg Dehrmann, »Die Idee der Universität. Friedrich August Wolf und die Praxis des Seminars«, Zeitschrift für Ideengeschichte 5/1 (2011), 105-117; Carlos Spoerhase, »Gegen Denken? Über die Praxis der Philologie«, Deutsche Vierteljahrsschrift für Literaturwissenschaft und Geistesgeschichte 89 (2015), 637-646.

107 Zur Etablierung der Atomphysik vgl. Kapitel 1 in Eckert (Anm. 5).

108 Konkret befassten sich die Atomphysiker gerade in der Anfangsphase der Quantenphysik zumeist mit Spektren - so hatten etwa bestimmte experimentell registrierte »Unregelmäßigkeiten« bei der Schwarzkörperstrahlung, die klassisch nicht erklärbar waren, Max Planck und Albert Einstein zu Beginn des Jahrhunderts zu neuen Konzeptionen (Planck'sches Wirkungsquantum, photoelektrischer Effekt) angeregt; Werner Heisenberg formulierte ca. 20 Jahre später seine Matrizenmechanik im Blick auf jeweils messbare Differenzen von Strahlungsfrequenzen und Spektrallinienintensitäten.

109 Vgl. dazu Christian Benne, Nietzsche und die historisch-kritische Philologie, Berlin, New York 2005, hier: 197-202. 
alle Texte $\ll^{110}$ wird, ernst, dann ist von einer charakteristischen, philologischen Prägung der Wissenskultur der deutschsprachigen theoretischen Physik am Beginn des 20. Jahrhunderts auszugehen. In diese Richtung weist auch eine Bemerkung von Hans Ulrich Gumbrecht, der in seiner Schrift Die Macht der Philologie gerade »die Forschungstätigkeit im Bereich der theoretischen Physik « und das »(beispielsweise $>$ philologische $<$ ) Nachdenken über ein vorsokratisches Fragment « miteinander in Beziehung setzt. ${ }^{111}$ Doch mehr noch: Die im vorliegenden Aufsatz am Beispiel Erwin Schrödingers herausgestellten Zusammenhänge legen nahe, dass die interdisziplinäre Verschaltung von theoretischer Physik und klassischer Philologie im frühen 20. Jahrhundert über einen solchen, eher intuitiv formulierten Konnex hinausreicht. Auch wenn die Physik die Philologie als Leitwissenschaft um 1900 abgelöst haben mag, konnte deren Macht noch lange ins 20. Jahrhundert nachwirken, und zwar nicht nur als Relikt und Referenzfeld einer überkommenen humanistischen Gelehrtentradition. ${ }^{12}$ Der zeitgemäße theoretische Physiker trat mitunter eher als Text- denn als klassischer Naturforscher auf. Er empfand sich selbst nicht mehr nur als Leser im mannigfaltigen Buch der Natur, sondern zunehmend auch als dessen Editor, der aus vielen vermeintlich zusammenhanglosen Fragmenten ein sinnvolles Ganzes zu generieren suchte.

Förderung Die Arbeit an diesem Aufsatz wurde gefördert durch ein Erwin Schrödinger-Stipendium des Austrian Science Fund (FWF), Projekt-Nr. J4175-G30 (Magdalena Gronau), sowie durch ein Initialisierungsstipendium der Universität Erfurt (Martin Gronau).

Funding Open Access funding enabled and organized by Projekt DEAL.

Open Access Dieser Artikel wird unter der Creative Commons Namensnennung 4.0 International Lizenz veröffentlicht, welche die Nutzung, Vervielfältigung, Bearbeitung, Verbreitung und Wiedergabe in jeglichem Medium und Format erlaubt, sofern Sie den/die ursprünglichen Autor(en) und die Quelle ordnungsgemäß nennen, einen Link zur Creative Commons Lizenz beifügen und angeben, ob Änderungen vorgenommen wurden.

Die in diesem Artikel enthaltenen Bilder und sonstiges Drittmaterial unterliegen ebenfalls der genannten Creative Commons Lizenz, sofern sich aus der Abbildungslegende nichts anderes ergibt. Sofern das betreffende Material nicht unter der genannten Creative Commons Lizenz steht und die betreffende Handlung nicht nach gesetzlichen Vorschriften erlaubt ist, ist für die oben aufgeführten Weiterverwendungen des Materials die Einwilligung des jeweiligen Rechteinhabers einzuholen.

Weitere Details zur Lizenz entnehmen Sie bitte der Lizenzinformation auf http://creativecommons.org/ licenses/by/4.0/deed.de.

\footnotetext{
110 Pollock (Anm. 78), hier: 43.

111 Hans Ulrich Gumbrecht, Die Macht der Philologie. Über einen verborgenen Impuls im wissenschaftlichen Umgang mit Texten, Frankfurt a.M. 2003. Das verbindende Element, so Gumbrecht, sei das »ästhetische Erlebnis«, dem »jede Form wissenschaftlicher Arbeit [...] nahestünde« - eine Behauptung, der Schrödinger, der in seinem Aufsatz »Wissenschaft - Kunst - Spiel « eine Wesensverwandtschaft zwischen den drei titelgebenden Sphären postuliert hat, vermutlich zustimmen würde. Vgl. Erwin Schrödinger, »Wissenschaft - Kunst - Spiel«, Die Koralle 6 (1930), 404, 410, 425-426.

112 Vgl. auch Andrea Albrecht, »Das Konzept des Klassischen in der modernen Naturwissenschaft und Mathematik. Wilhelm Ostwald, Oswald Spengler und Max Bense «, in: Thorsten Valk (Hrsg.), Die Rede vom Klassischen. Transformationen und Kontinuitäten im 20. Jahrhundert, Göttingen 2020, 251-276.
} 